\title{
SYNGAP1 Controls the Maturation of Dendrites, Synaptic Function, and Network Activity in Developing Human Neurons
}

\author{
Nerea Llamosas, ${ }^{1}{ }^{\oplus}$ Vineet Arora, ${ }^{1}$ Ridhima Vij,,${ }^{2,3}$ Murat Kilinc, ${ }^{1}$ Lukasz Bijoch, ${ }^{4}$ Camilo Rojas, ${ }^{1}$ Adrian Reich, ${ }^{5}$ \\ BanuPriya Sridharan, ${ }^{6}$ Erik Willems, ${ }^{7}$ David R. Piper, ${ }^{7}$ Louis Scampavia, ${ }^{6}$ Timothy P. Spicer, ${ }^{6}$ \\ Courtney A. Miller, ${ }^{1,6}$ J. Lloyd Holder, ${ }^{2,3}$ and Gavin Rumbaugh ${ }^{1}$ \\ ${ }^{1}$ Department of Neuroscience, Scripps Research, Jupiter, Florida $33458,{ }^{2}$ Jan and Dan Duncan Neurological Research Institute at Texas Children's Hospital, \\ Houston, Texas 77030, ${ }^{3}$ Department of Pediatrics, Baylor College of Medicine, Houston, Texas 77030, ${ }^{4}$ Laboratory of Neurobiology, BRAINCITY, Nencki \\ Institute of Experimental Biology, Polish Academy of Sciences, Warsaw, Poland, ${ }^{5}$ Center for Computational Biology and Bioinformatics, Scripps Research, \\ Jupiter, Florida 33458, ${ }^{6}$ Department of Molecular Medicine, Scripps Research, Jupiter, Florida 33458, and ${ }^{7}$ Cell Biology, Thermo Fisher Scientific, Carlsbad, \\ California 92008
}

SYNGAP1 is a major genetic risk factor for global developmental delay, autism spectrum disorder, and epileptic encephalopathy. De novo loss-of-function variants in this gene cause a neurodevelopmental disorder defined by cognitive impairment, social-communication disorder, and early-onset seizures. Cell biological studies in mouse and rat neurons have shown that Syngap1 regulates developing excitatory synapse structure and function, with loss-of-function variants driving formation of larger dendritic spines and stronger glutamatergic transmission. However, studies to date have been limited to mouse and rat neurons. Therefore, it remains unknown how SYNGAP1 loss of function impacts the development and function of human neurons. To address this, we used CRISPR/Cas9 technology to ablate SYNGAP1 protein expression in neurons derived from a commercially available induced pluripotent stem cell line (hiPSC) obtained from a human female donor. Reducing SynGAP protein expression in developing hiPSC-derived neurons enhanced dendritic morphogenesis, leading to larger neurons compared with those derived from isogenic controls. Consistent with larger dendritic fields, we also observed a greater number of morphologically defined excitatory synapses in cultures containing these neurons. Moreover, neurons with reduced SynGAP protein had stronger excitatory synapses and expressed synaptic activity earlier in development. Finally, distributed network spiking activity appeared earlier, was substantially elevated, and exhibited greater bursting behavior in SYNGAP1 null neurons. We conclude that SYNGAP1 regulates the postmitotic maturation of human neurons made from hiPSCs, which influences how activity develops within nascent neural networks. Alterations to this fundamental neurodevelopmental process may contribute to the etiology of SYNGAP1-related disorders.

Key words: autism; development; epilepsy; iPSC; synapse; SYNGAP1

Significance Statement

SYNGAP1 is a major genetic risk factor for global developmental delay, autism spectrum disorder, and epileptic encephalopathy. While this gene is well studied in rodent neurons, its function in human neurons remains unknown. We used CRISPR/Cas9 technology to disrupt SYNGAP1 protein expression in neurons derived from an induced pluripotent stem cell line. We found that induced neurons lacking SynGAP expression exhibited accelerated dendritic morphogenesis, increased accumulation of postsynaptic markers, early expression of synapse activity, enhanced excitatory synaptic strength, and early onset of neural network activity. We conclude that SYNGAP1 regulates the postmitotic differentiation rate of developing human neurons and disrupting this process impacts the function of nascent neural networks. These altered developmental processes may contribute to the etiology of SYNGAP1 disorders.

Received May 31, 2020; revised July 26, 2020; accepted Aug. 24, 2020.

Author contributions: G.R., N.L., V.A., M.K., A.R., E.W., D.R.P., L.S., T.P.S., C.A.M., J.L.H., and R.V. designed research; G.R., N.L., V.A., M.K., L.B., C.R., A.R., C.A.M., J.L.H., and R.V. analyzed data; G.R. wrote the first draft of the paper; G.R. and N.L. wrote the paper; N.L., V.A., M.K., L.B., C.R., A.R., B.S., E.W., and R.V. performed research; N.L., V.A., M.K., L.B., A.R., E.W., D.R.P., L.S., T.P.S., C.A.M., J.L.H., and R.V. edited the paper; E.W., D.R.P., L.S., and T.P.S. contributed unpublished reagents/analytic tools.

This work was supported in part by National Institute of Mental Health, National Institutes of Health Grants MH096847 and MH108408 to G.R., and the National Institute for Neurological Disorders and Stroke NS064079 and NS110307 to G.R. and NS091381 to J.L.H. N.L. was supported by SynGAP Research Fund postdoctoral training fellowship. J.L.H. was supported by SynGAP Research Fund, Doris Duke Charitable Foundation Grant 201763, the
Robbins Foundation, and Charif Souki. V.A. was supported by Leon and Friends postdoctoral training fellowship. L.B. was supported in part by Boehringer Ingelheim Fonds travel grant for junior researchers. hiPSC clones and data supporting the findings of this study are available from the corresponding author upon reasonable request.

B. Sridharan's present address: GlaxoSmithKline 1250 S. Collegeville Road, Collegeville, Pennsylvania 19426.

E.W. and D.R.P. are employed by Thermo Fisher Scientific. The remaining authors declare no competing financial interests.

Correspondence should be addressed to Gavin Rumbaugh at gavin@scripps.edu.

https://doi.org/10.1523/JNEUROSCl.1367-20.2020

Copyright $\odot 2020$ the authors 


\section{Introduction}

Pathogenic loss-of-function variants in the SYNGAP1 gene are causally linked to global developmental delay (GDD)/intellectual disability (ID) (Hamdan et al., 2009; Rauch et al., 2012; Deciphering Developmental Disorders Study, 2015, 2017) and severe epilepsy (Carvill et al., 2013; von Stulpnagel et al., 2015; Vlaskamp et al., 2019). SYNGAP1 is also strongly implicated in autism spectrum disorders (Rauch et al., 2012; O'Roak et al., 2014; Satterstrom et al., 2020). While pathogenic variants in SYNAGP1 are overall rare, they are common relative to the pool of genes capable of causing sporadic neurodevelopmental disorders, explaining up to $\sim 1 \%$ of GDD/ID cases (Berryer et al., 2013; Parker et al., 2015), which is in the range of other monogenic disorders that are more extensively studied by the scientific community. Causality of SYNGAP1 pathogenicity is now clear because of its high intolerance to loss-of-function mutations. The constraint metric loss-of-function observed/expected upper bound fraction is 0.05 derived from the $>141,000$ individuals from the version 2.1.1 gnomAD database (Karczewski et al., 2020), demonstrating its extreme loss-of-function mutation intolerance. Moreover, the non-neuro dataset of exomes from $>114,000$ individuals from gnomAD reveals only three frameshift variants with two of these lying in the extreme five or three prime regions of the gene, an area known to undergo extensive alternative splicing. Based on substantial clinical evidence, proper SYNGAP1 expression is required for normal human brain development and function.

Syngap1 gene function has been studied in rodent neurons (Kilinc et al., 2018; Gamache et al., 2020), where it is a potent regulator of Hebbian plasticity at excitatory synapses. Heterozygous $\mathrm{KO}$ mice exhibit deficits in hippocampal LTP evoked through a variety of synaptic stimulation protocols (Komiyama et al., 2002; Kim et al., 2003). Genetic reexpression of Syngap1 in adult mutant mice rescues hippocampal LTP and associated downstream signaling pathways (Ozkan et al., 2014). Thus, SynGAP regulation of synapse plasticity is a dynamic function of the protein that is retained throughout life. Hundreds of genes regulate synaptic plasticity as referenced by the Gene Ontology browser (http://www.informatics.jax.org/vocab/gene_ontology/ GO:0048167). However, most of them do not cause disease when heterozygously expressed, as is the case for SYNGAP1. Therefore, SYNGAP1 likely has additional functions beyond regulation of synapse plasticity that contribute to disease etiology. Indeed, SynGAP expression in developing mouse neurons acts to regulate the maturation rate of excitatory synapse strength, and this function is independent from its role in plasticity. SynGAP protein expression rises quickly during postnatal development (Gou et al., 2020), and its expression during this period is critical for shaping the strength of nascent excitatory synapses (Clement et al., 2012, 2013). In contrast to Hebbian processes, this function of rodent Syngap1 is linked to biological processes unique to developing neurons. Enhanced baseline excitatory synaptic strength in hippocampal neurons is transiently observed during the first 3 postnatal weeks of brain development, and inducing heterozygosity of Syngap1 beyond this period has minimal effect on resting synaptic function in these neurons (Clement et al., 2012).

The understanding of how this gene contributes to diseaserelevant biology is limited because information on its function in human neurons is lacking. This is limiting because there are fundamental differences in how human and rodent brains develop. For example, humans express neoteny, or slowing of development, which is thought to promote an extended period of neural network refinement that promotes higher cognitive functions. An example of neoteny at the neurobiological level is the relative pace of human neuron development compared with rodents (Petanjek et al., 2011; Charrier et al., 2012), with human neurons exhibiting a much slower pace of postmitotic differentiation. Given that Syngap1 alters measures of neuronal maturation in rodents (Clement et al., 2012, 2013; Aceti et al., 2015), this function of the gene may be amplified in slower developing human neurons. To test this idea, we created a SYNGAP1 KO humaninduced pluripotent stem cell (hiPSC) line using CRISPR/ Cas9 technology and measured various parameters of neuronal maturation.

\section{Material and Methods}

\section{Maintenance of hiPSC cultures}

All hiPSC work was performed in accordance with approved protocols from appropriate Institutional Review Boards. All products were purchased from Thermo Fisher Scientific unless otherwise noted. All studies were performed in a single human hiPSC line obtained from a female donor. The stable human episomal Cas9 hiPSC cell line was obtained from Thermo Fisher Scientific (A33124) and was expanded according to the manufacturer's suggested protocol. This line was previously used for generating neurons (Sridharan et al., 2019). Briefly, culture plates were coated with Vitronectin-N (A14700), diluted 1:100 in DPBS (14190094), and incubated at $37^{\circ} \mathrm{C}$ for at least $1 \mathrm{~h}$ before hiPSC plating. Cryopreserved hiPSC cells were gently thawed in a $37^{\circ} \mathrm{C}$ water bath and transferred to a $15 \mathrm{ml}$ conical tube with Complete hiPSC Medium $+1 \%$ RevitaCell supplement (A2644501). Cells were then centrifuged at $200 \times g$ for $4 \mathrm{~min}$, and the hiPSC pellet was resuspended in fresh medium and plated on vitronectin coated flasks. Twenty-four hours later, cells were switched and maintained in Complete Stemflex Medium (w/o RevitaCell) with daily medium changes until $70 \%$ confluent. Cells were then harvested with TrypLE Select (12563011) and further maintained or plated for experimental purposes. For limiting dilution cloning, hiPSCs were plated in 96-well plates coated with $2.5 \mu \mathrm{m} / \mathrm{ml}$ rhLaminin-521 (A29248).

\section{Generation of SYNGAP1 KO hiPSC lines}

Guide RNA ( $g$ RNA) sequences targeting exon 7 of SYNGAP1 were selected using the Zhang laboratory CRISPR design tool (http://zlab.bio/guidedesign-resources) and acquired from IDT in single guide RNAs ( $g$ RNA) format. Cas9-hiPSCs were transfected with sgRNAs by using Lipofectamine CRISPRMAX (Thermo Fisher Scientific, CMAX00001) according to the manufacturer's instructions. Editing efficiency of individual $s g$ RNAs was determined using GeneArt Genomic Cleavage Detection Kit (Thermo Fisher Scientific, A24372). sgRNA-5 (target sequence $5^{\prime}$-TCTTTCGGCCGCAGACCGAC-3') demonstrated the highest efficiency and was selected for downstream applications. To generate the SYNGAP1 KO hiPSC lines, cells were transfected with $s g$ RNA-5. Twenty-four hours after the transfection, cells were plated in rhLaminin-521-coated 96-well plates with an average density of 0.5 cells/well. Colonies derived from a single cell were expanded and cryopreserved with Recovery cell culture freezing medium (Thermo Fisher Scientific, 12648010). Approximately 70 colonies originating from a single cell were analyzed for indels around the sgRNA targeting site. Multiple clones with either unedited (WT) or edited sequences were isolated and expanded. Potential KO clones were identified by Sanger sequence traces and prioritized for downstream validation 
studies. Pluripotency of individual clones was confirmed via TaqMan Array Human Stem Cell Pluripotency Panel (4385344) according to the manufacturers' instructions. Each expanded clone was tested, and confirmed negative, for mycoplasma contamination using Universal Mycoplasma Detection Kit (ATCC, 30-1012K).

Whole exome sequencing (WES)

Genomic DNA from the four experimental clones and a sample of the original Cas9 hiPSC line (before CRISPR transfection) were extracted using PureLink Genomic DNA mini Kit (Invitrogen, \#k1820-02) using included instructions. Genomic DNA from each of the five samples was shipped to HudsonAlpha Institute for Biotechnology, Genome Sequencing Center for WES.

Library preparation and quality control. DNA samples were normalized to $1000 \mathrm{ng}$ of DNA in $50 \mu \mathrm{l}$ of water. Following normalization, samples were acoustically sheared via Covaris LE-220 instrument to a final fragment size of $\sim 350-400 \mathrm{bp}$. The sheared DNA was then transformed into a standard Illumina paired-end sequencing library via standard methods. The sheared DNA was end-repaired and A-tailed using Roche-Kapa End-Repair and ATailing kits under the manufacturer's recommended conditions. Standard Illumina paired-end adaptors were ligated to the Atailed DNA. Following ligation, the reactions were purified using AMPure XP beads. The purified ligated DNA was amplified via PCR using Roche KAPA HIFI PCR reagents using four cycles of PCR. The primers used in the PCR step introduced 8-base, unique, dual indexes in the i5 and i7 positions to allow sample identification/demultiplexing following sequencing. The final library was quality controlled using size verification via PerkinElmer LabChip GX and real-time PCR using the Roche KAPA SYBR FAST qPCR Master Mix, primers, and standards according to the manufacturer's directions. Libraries were normalized to $1.4 \mathrm{~nm}$ stocks for use in clustering and sequencing.

IDT exome capture and quality control. After library construction, samples were multiplexed for capture at 5 samples per pool with each sample contributing between 200 and $300 \mathrm{ng}$ to each pool. Pooled samples were purified with beads and eluted in a volume of $30 \mu \mathrm{l}$. Pooled samples were hybridized with the NimbleGen SeqCap EZ Exome v3 probes with minor modifications for automation. Briefly, multiplexed samples were dried down in the presence of COT-1 and a blocker mix for $1.5 \mathrm{~h}$. Libraries were then resuspended in a mix of hybridization buffer and baits. Libraries were hybridized overnight at $65^{\circ} \mathrm{C}(72 \mathrm{~h})$. Post-hybridization takedown occurred 72 h later. Briefly, captured libraries were bound to streptavidin beads. Once bound, washing occurred per the manufacturer's recommendations. Final elution of captured libraries was in $20 \mu \mathrm{l}$ of nuclease-free water. Libraries were amplified with six cycles of PCR and a final bead purification. Post-hybridization exome concentrations were measured via Picogreen, and library sizes were determined via the LabChip GX Touch HT (PerkinElmer). Additionally, libraries were quantitated with real-time PCR using the KAPA Library Quantification Kit (Roche) per the manufacturer's instructions to determine final library nanomolarity. Final exome libraries were pooled at a concentration of $1.8 \mathrm{~nm}$. The pooled exome libraries were distributed across four lanes on an S4 flow cell and sequenced using $150 \mathrm{bp}$ paired-end approach on a NovaSeq 6000 instrument (Illumina). All sequencing was performed on the Illumina NovaSeq 6000 platform by loading a pool samples to the equivalent loading of 24 samples per flow cell. Following sequencing, all base-calling was performed using standard Illumina software to generate the final FASTQ files for each sample. Following sequencing, all base-calling was performed using standard Illumina software to generate the final FASTQ files for each sample. Alignment and variant calling were performed with BWA, samtools, and GATK to verify coverage and performance. Samples yielded a minimum of $440 \mathrm{M}$ paired reads at $150 \mathrm{nt}$ read length with a mean coverage of $>30 \times$.

\section{Karyotyping}

Karyotyping was performed as previously described (Sridharan et al., 2019). Briefly, differentiated induced neurons (iNeurons) were assessed for any chromosomal aberrations using the Karyostat assay (Thermo Fisher Scientific).

\section{Generation of iNeurons from Cas9-hiPSC single-cell clones}

Ngn2 transcription factor-induced iNeurons were generated as previously described (Sridharan et al., 2019) with minor modifications. Briefly, Cas9-hiPSCs were harvested using TrypLE Select, and 2 million cells were plated on vitronectin-coated T75 flask on day 1 . On day 2, medium was removed, and an appropriate amount of lentivirus-expressing Ngn2 (Addgene, 52047) and rtTA (Addgene, 20342) were administered in Complete Stemflex Medium, including 1\% RevitaCell (MOI 2 for both lentivirus). After $24 \mathrm{~h}$, the medium was aspirated and replaced with Induction Media-induced TetO gene expression. The next day, medium was refreshed with Induction Media $+2 \mu \mathrm{g} / \mathrm{ml}$ puromycin (A1113803), which was included for selection of iNeurons. Twenty-four hours later, iNeurons were harvested using Accutase (A1110501) and plated on PDL-coated plates in iNeuron Maintenance Media: Neurobasal (211103049) + 1\% GlutaMax (35050061) + 2\% B27 (17504044) + 10 ng/ml BDNF $(\mathrm{PHC} 7074)+10 \mathrm{ng} / \mathrm{ml}$ GDNF $(\mathrm{PHC} 7036)+10 \mathrm{ng} / \mathrm{ml}$ NT3 $(\mathrm{PHC} 7045)+2.5 \%$ FBS (10082139) (all from Thermo Fisher Scientific) $+10 \mu \mathrm{g} / \mathrm{ml} \mathrm{FuDR} \mathrm{(Sigma} \mathrm{Millipore,} \mathrm{F0503)} \mathrm{along}$ with primary rat glia (neuron/glia ratio 2.5/1). Half of the medium was changed with fresh iNeuron Maintenance Media every $4-5 \mathrm{~d}$.

\section{Dendritic tracing}

Each well of a 96-well imaging plate contained $\sim 50,000$ cells per well, consisting of $\sim 32,000$ iNeurons $+\sim 18,000$ primary rat astrocytes along with $0.1 \%$ ( $\sim 50$ per well) of eGFP-positive human-iNeurons derived from the same clone. eGFP-positive iNeurons were created through a separate induction as stated above, except that an additional lentivirus expressing eGFP under the control of a TET-responsive promoter was included (Addgene, catalog \#30130). eGFP-positive neurons were mixed with eGFP-negative neurons in the 96-well plates. iNeurons derived from either of the WT or KO clones were compared by tracing primary (originating from the soma), secondary, and tertiary dendrites, as well as total dendrite length. Tracing data were obtained by imaging live iNeurons at DIV45 with an InCell Analyzer 6000 automated confocal microscope $(20 \times$ magnification). A sample of 30 randomly selected neurons per genotype ( $n=3$ per well $\times 10$ wells in a 96 -well plate) was selected, and then dendrites were traced with the Simple Neurite Tracer software plugin distributed by Fiji-ImageJ. Data represent the average lengths in microns for all subtypes of dendrites.

\section{Immunocytochemistry}

iNeurons were replated, along with primary rat astrocytes, at a density of iNeurons 200,000/120,000 astrocytes per well, on 15 $\mathrm{mm}$ cover glass coated with PDL/fibronectin (Neuvitro, GG-15- 
fibronectin), in 24-well plates. At DIV30-DV45, cells were fixed and labeled with primary antibodies: anti-PSD95 (mouse-raised; Abcam, catalog \#ab2723, $100 \mu \mathrm{g}$ ), anti-GluA1 (rabbit-raised; Cell Signaling Technology, catalog \#13185S), and anti-MAP2 (guinea pig-raised; Synaptic Systems, catalog \#188004). Then, secondary antibodies were applied (goat anti-mouse Alexa-488, Abcam, catalog \#ab150113, $500 \mu \mathrm{g}$; goat anti-rabbit Alexa-568, Thermo Fisher Scientific, catalog \#A11036, goat anti-guinea pig Alexa-647, Thermo Fisher Scientific, catalog \#A21450). Images of neurons from multiple coverslips per culture were taken under UPlanSApo $100 \times 1.4 \mathrm{NA}$ oil-immersion objective mounted on Olympus FV1000 laser-scanning confocal microscope (1 image $=1$ FOV). Neuronal somas from individual FOV were manually calculated based on raw MAP2 signals. Total area of MAP2/FOV was determined on the area of mask of MAP2 signal. Number of detected particles of GLUA1 and PSD95 per FOV was determined based on threshold-based signal masks. Thresholds were kept constant across all images.

\section{Immunoblotting}

iNeurons were cocultured with rat glia $(500,000$ iNeurons, 100,000 glia) seeded on 12-well plates. After 30-60 d in culture, media was removed and the wells were washed with PBS, after which the PBS was replaced with $200 \mu$ l of RIPA buffer (Cell Signaling Technology) containing Phosphatase Inhibitor Cocktails 2 and 3 (Sigma Millipore) and MiniComplete Protease Inhibitor Cocktail (Roche Diagnostics), the wells were scraped using a sterile cell scraper on each well, transferred to tubes in dry ice, and stored at $-80^{\circ} \mathrm{C}$. After thawing on ice, samples were sonicated using a probe sonicator 5 times with $2 \mathrm{~s}$ pulses. Sample protein levels were measured (Pierce BCA Protein Assay Kit, Thermo Fisher Scientific), and volumes were adjusted to normalize microgram per microliter protein content. A total of $10 \mu \mathrm{g}$ of protein per sample was loaded and separated using SDS-PAGE on 4\%-15\% gradient stain-free Tris-glycine gels (Mini Protean TGX, Bio-Rad), after which gels were removed from their casing and activated using a UV transilluminator for $1 \mathrm{~min}$ to visualize total protein levels following Bio-Rad product instructions. The gels were transferred to low fluorescence PVDF membranes $(45 \mu \mathrm{m})$ with the Power Blotter Semi-dry Transfer System (Thermo Fisher Scientific). Following transfer, total protein levels were obtained by taking an image of the membranes using the ChemiDoc imaging system (Bio-Rad). Membranes were blocked with $5 \%$ powdered milk in buffer and probed with pan-SynGAP (1:1000, \#5539, Cell Signaling) or SynGAP- $\alpha 2$ (abcam, ab77235), overnight at $4^{\circ} \mathrm{C}$ and HRP-conjugated anti-rabbit antibody (1:2000, W4011, Promega) for $1 \mathrm{~h}$ at room temperature followed by ECL signal amplification and chemiluminescence detection (SuperSignal West Pico Chemiluminescent Substrate; Thermo Fisher Scientific). Blot band densities were obtained using the ChemiDoc imaging system (Bio-Rad). SynGAP levels of immunoreactivity and total protein levels were assessed by densitometric analysis of generated images with ImageJ. SynGAP values were normalized to total protein levels obtained from the membranes before antibody incubations.

\section{Whole-cell electrophysiology}

iNeuron measurements were performed up to DIV50 as described with minor modifications (Sridharan et al., 2019). For current studies, iNeurons were cocultured with cryo-recovered primary rat astrocytes (seeded at 20,000 iNeurons $+10,000$ astrocytes per well) in 24 -well plate on $15 \mathrm{~mm}$ coverslips. The cocultures were maintained in plating medium and additionally supplemented with $10 \mu \mathrm{g} / \mathrm{ml}$ FUDR (catalog \#F0503, Sigma
Millipore). For whole-cell recordings, intrinsic electrical properties were inspected immediately after gaining access to the cell, and miniature excitatory synaptic currents were recorded in the presence of TTX $(0.5 \mu \mathrm{M})$ at room temperature in voltage-clamp configuration (cells were held at $-60 \mathrm{mV}$ with a Multiclamp 700B amplifier, Molecular Devices). The bath solution contained the following (in mM): $140 \mathrm{NaCl}, 5 \mathrm{KCl}, 2 \mathrm{CaCl}_{2}, 2 \mathrm{MgCl}_{2}, 10$ HEPES-NaOH, and 10 glucose, $\mathrm{pH}$ to 7.4 adjusted with $\mathrm{NaOH}$. Pipettes pulled from borosilicate glass capillary tubes (catalog \#G85150T-4, Warner Instruments) using a P-97 pipette puller (Sutter Instrument) were filled with the following intracellular solution (in $\mathrm{mm}$ ): $123 \mathrm{~K}$-gluconate, $10 \mathrm{KCl}, 1 \mathrm{MgCl}_{2}, 10 \mathrm{HEPES}$ $\mathrm{KOH}, 1$ EGTA, $0.1 \mathrm{CaCl}_{2}, 1 \mathrm{~K}_{2}$-ATP, $0.2 \mathrm{Na}_{4}$-GTP, and 4 glucose, $\mathrm{pH}$ adjusted to 7.4 with $\mathrm{KOH}$. Resistance of the pipettes filled with the intracellular solution was between 3 and $5 \mathrm{~m} \Omega$. Series resistance was monitored without compensation with $5 \mathrm{mV}$ depolarizing steps $(200 \mathrm{~ms})$ induced every $60 \mathrm{~s}$ to ensure sufficient and stable electrical access to the cell. Data were sampled at $10 \mathrm{kHz}$, post hoc filtered, and analyzed offline using Clampfit (Molecular Devices). Single-peak mEPSCs were detected using a semiautomated sliding template detection procedure in which the template was generated by averaging multiple spontaneous currents. Each detected event was visually inspected and discarded if the amplitude was $<7 \mathrm{pA}$.

\section{Microelectrode array (MEA) analysis}

Cell culture and neural progenitor cell (NPC) differentiation. Individual SYNGAP1 WT and KO hiPSC clones were maintained on Matrigel-coated plates in Stem Flex media (Thermo Fisher Scientific). NPCs were differentiated from hiPSCs using a dual SMAD inhibition protocol (Jiang et al., 2017). Briefly, stem cell lines were dissociated using Accutase, and embryoid bodies were generated from the stem cell lines in the Aggrewells using neural proliferation medium along with BMP and WNT inhibitors (dorsomorphin $4 \mu \mathrm{M}$ and SB-431542:SB $10 \mu \mathrm{M}$; Sigma Millipore), administered on day 2 of neural induction. Around day 5, EBs were gently collected and plated on Matrigel-coated plates for the formation of rosettes. To promote dorsalization, $10 \mu \mathrm{M}$ cyclopamine (Stem Cell technologies) was added to the plates starting day 6. Both inhibitors and cyclopamine were added to the media until day $\sim 9$. Rosettes were collected between day 14 and day 16 and plated on gelatin-coated plates so that the non-neural cells were preferentially removed from floating neural progenitors, which were then dissociated to form a monolayer culture of NPCs. NPCs were grown and expanded on Matrigel-coated plates before the cells were plated directly on a MEA plate for neuronal differentiation.

MEA analysis and neuronal differentiation. We used an MEA system (Axion Biosystems) to perform neurophysiological characterization of iNeurons. Neuronal differentiation of NPCs was performed directly on MEA plates; $1.6 \times 10^{4}$ NPCs suspended in a $5 \mu \mathrm{l}$ droplet of neural precursor medium were plated as on top of a 16-electrode array (area $\sim 1 \mathrm{~mm}^{2}$ ) inside a single well of 48 -well MEA plate pretreated with $0.1 \%$ PEI solution prepared in borate buffer, $\mathrm{pH}$ 8.4. Two days later, neuronal differentiation was initiated using a neuronal induction medium (prepared from equal volumes of DMEM/F12 and neurobasal medium without growth factors) prepared in-house. Neuronal induction medium was exchanged every other day for $7 \mathrm{~d}$. Differentiation of the NPCs into forebrain cortical neurons was performed using previously established neuronal differentiation medium, NDM, which includes a cocktail of differentiation factors (BDNF, GDNF, NT-3, dibutyryl-cAMP, ascorbic acid) 
(Jiang et al., 2017). After differentiation, NDM was replaced with BrainPhys for further maturation (Stem Cell Technologies), and neurons were cultured for at least 1 week before neuronal activity was recorded. Neuronal activity was recorded continuously for 5 min from the multiwell MEA plate each week until 6 weeks of neuronal maturation after differentiation. Field potential changes were recorded and analyzed using Axis Navigator and Axis metric plotting software (Axion Biosystems). Temporal raster plots were generated using Neural Metric Tool software (Axion Biosystems). For data analysis, a burst was identified as a group of at least 5 spikes, separated by an interspike interval of $<100$ ms. Network bursts were defined as a minimum of 50 spikes with a maximum interspike interval of $100 \mathrm{~ms}$ covering at least $35 \%$ of electrodes in a well.

\section{Statistics}

GraphPad Prism 8 software was used for all statistical analyses. All data were tested for normality. Accordingly, parametric or nonparametric tests were applied. For tracing data analyses, clonal comparisons were performed using Kruskal-Wallis test followed by Dunn's multiple comparison test. For genotype comparisons, Mann-Whitney tests were applied. For immunostaining experiments, Mann-Whitney $U$ tests or unpaired two-tailed $t$ tests were used. For clonal comparisons of electrophysiological data Kruskal-Wallis followed by corrected Dunn's multiple comparison tests or one-way ANOVA followed by Tukey tests were used. Statistical differences of percentage mEPSC-expressing neurons were determined by Fischer exact test pairwise comparisons. For genotypic comparisons of whole-cell electrophysiological data, Mann-Whitney $U$ tests, or unpaired two-tailed $t$ tests were performed. When comparing cumulative probability data between clones or genotypes the Kolmogorov-Smirnov test was used. For MEA studies, statistical analyses were performed using two-way repeated-measures ANOVA. Non-normal data throughout the text are presented as box-and-whisker plots where the middle, boxes, and whiskers represent the median, interquartile range, and min to max, respectively. Normally distributed data are presented as mean \pm SEM. Differences were considered to be significant for $p<0.05$. Exact $p$ values are reported when provided by the software.

\section{Results}

SYNGAP1 patients are heterozygous for deleterious variants, largely causing haploinsufficiency. Given that the penetrance of deleterious pathogenic SYNGAP1 variants is $\sim 100 \%$, this gene is critically important for human brain development. Thus, it is important to define the functions of the gene in developing human neurons. To begin to define these functions, we sought to create a SYNGAP1 null hiPSC model because this would be expected to induce the strongest possible phenotypes in developing iNeurons. To create SYNGAP1 null hiPSCs, we performed CRISPR editing of a common exon within the human locus. Exon 7 was targeted (Fig. 1A) for nonhomologous end joining repair for the following reasons: (1) it is a common exon present in most, if not all, SYNGAP1 transcripts (McMahon et al., 2012; Gou et al., 2020); (2) it is downstream of multiple stop-gain or small indel patient-specific variants (Jimenez-Gomez et al., 2019); and (3) targeting it in other species results in ablation of SynGAP protein (Kim et al., 2003; Clement et al., 2012). Four single-cell clones were identified and selected for downstream analysis. These clones contained either an edited (KO: clone \#4 and \#38) or unedited (WT: clone \#6 and \#30) exon 7 (Fig. 1B).
For clone \#4, the Sanger trace suggested distinct, but neighboring, single base pair deletions on each copy of SYNGAP1. This hypothesis was supported by WES, where $\sim 50 \%$ of the mapped reads contained a single base deleted, and the remaining reads had the neighboring base deleted (Fig. 1C). Combining WES with Sanger sequencing clarified CRISPR-mediated mutations in clone \#38. The Sanger trace suggested that there was a single 8 bp deletion near the CRISPR target site (Fig. 1B). However, WES demonstrated $\sim 50 \%$ fewer reads within exons 6 and 7, specifically in clone \#38 (Fig. 1D). This was suggestive of a large deletion that encompassed exons 6 and 7 , but was $<4 \mathrm{~kb}$ in size because exons 5 and 8 remained intact. Genomic PCR in this region was successful but failed to detect a band shift (Fig. 1E), although PCR amplification in this region was limited to $\sim 2.2$ $\mathrm{kb}$. Thus, for clone \#38, there were also likely two distinct deletions in each of the SYNGAP1 copies. With the targeted deletions mapped, we next assessed off-target effects of CRISPR. With the exception of the large deletion in clone \#38, in-depth mapping of the whole SYNGAP1 gene revealed that SYNGAP1 exon structure was largely intact across the four clones (Fig. $1 F$ ). Other than the targeted mutations around the CRISPR-targeted region, we observed very little genetic drift across the protein coding portion of the genome within the four clones. We observed only a few high confidence exonic small indels in each of the four CRISPR clones (Table 1). None of these indels was shared within the same gene, and none of them was homozygous. Thus, unbiased read-mapping of WES identified the sequences used to select the two "KO" clones, and these sequences appeared to be the most significant deviations among the four clones (Fig. 1G). Therefore, these four clones are essentially isogenic, with the exception of the targeted mutations used to select the two "KO" clones. Finally, karyotyping analysis (Fig. $1 H$ ) revealed no large alterations to chromosomal structure in any of the four clones, each of the clones passed self-renewal and pluripotency checks, and all tested negative for mycoplasma contamination.

Analysis of CRISPR targeting indicated that both "KO" clones contained distinct frameshift deletions in both copies of SYNGAP1 (Fig. 2A,B). This would be expected to induce nonsense-mediated decay of SYNGAP1 transcripts and corresponding reductions in SynGAP protein expression. To test this prediction, glutamatergic neurons were produced from each of the four clones using the Ngn2 induction method and then cocultured with rat astrocytes to induce functional synapses (Zhang et al., 2013). After $\sim 30-60 \mathrm{~d}$ of neuronal development, samples were immunoblotted for SynGAP protein levels. As predicted, neurons derived from both "KO" clones had significantly lower levels of SynGAP protein than "WT" clones. Reduced SynGAP signal was observed with antibodies recognizing either a core region of the protein (Pan-SynGAP) or to the C-terminus of a specific splice variant $(\alpha 2$; Fig. $2 C, D ; \mathrm{H}=12.29, p=0.0001$; WT\#6 vs KO\#4: $p=0.1876$; WT\#6 vs WT\#30: $p>0.9999$; WT\#6 vs KO\#38: $p=0.0140$; KO\#4 vs WT\#30: $p=0.5258$; KO\#4 vs KO\#38: $p>0.9999$; WT\#30 vs KO\#38: $p=0.0561, n=4$ per group). There was some residual SynGAP protein observed in samples derived from both $\mathrm{KO}$ clones, which may stem from low levels of SynGAP present in rat primary astrocytes. However, given that SynGAP signal is $<10 \%$ of control levels, the two KO clones appeared to produce iNeurons with nominal SynGAP protein expression.

Syngap1 loss-of-function in rodent neurons disrupts the maturation rate of dendrites and synapses. Therefore, we examined dendritic morphogenesis in developing iNeurons produced from each of the four human iPSC clones. Dendritic morphology was 

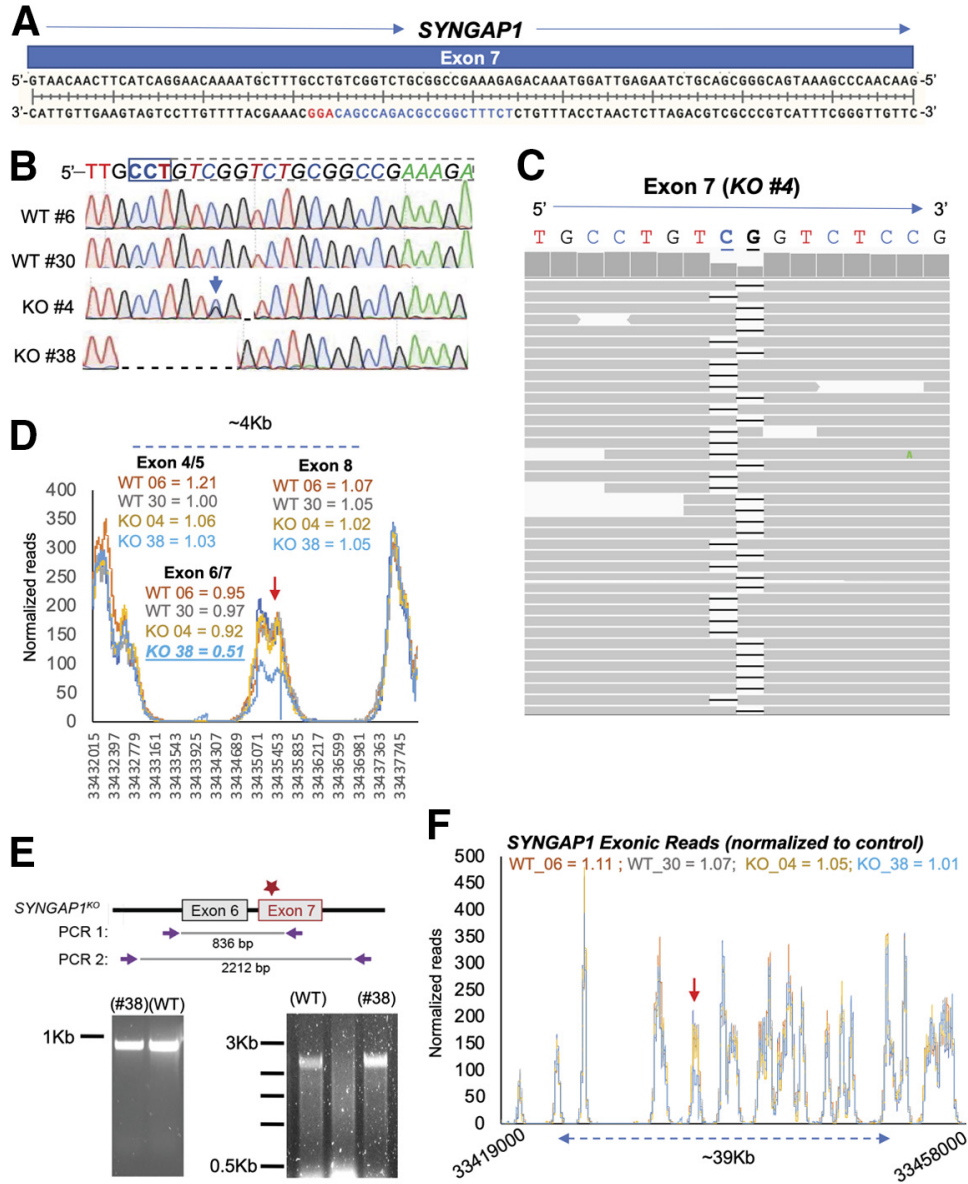

G

\begin{tabular}{|c|c|c|c|c|}
\hline Sample & $\begin{array}{l}\frac{\text { Total }}{\text { Indel }} \\
\text { count }\end{array}$ & $\begin{array}{l}\frac{\text { Indel }}{\text { count }} \\
\text { cabove } \\
\underline{0.8}\end{array}$ & $\begin{array}{l}\text { Location } \\
\text { (chrom:loc) }\end{array}$ & 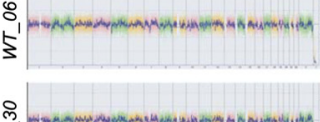 \\
\hline WT_06 & 7 & 0 & NA & \\
\hline WT_30 & 10 & 0 & NA & 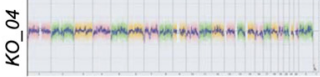 \\
\hline KO_38 & 13 & $1^{*}$ & 6:33435544 & 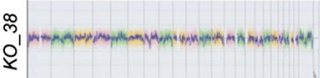 \\
\hline
\end{tabular}

Figure 1. Development of isogenic SYNGAP1 KO hiPSCS. A, Diagram represents CRISPR targeting within exon 7 (complete coding sequence) of the SYNGAP1 gene. Blue sequence represents the sgRNA. Red sequence represents the PAM site. $\boldsymbol{B}$, Sanger sequencing for two WT and two SYNGAP1 mutant clones derived from the CRISPR experiment. $\boldsymbol{C}$, Individual WES paired-end reads from clone \#4 near the CRISPR targeted region within SYNGAP1. D, Normalized mapped reads for the same samples around the Cas9 target sequence. $\boldsymbol{E}$, Genomic PCR to amplify DNA sequence flanking the CRISPR target site. $\boldsymbol{F}$, Normalized mapped reads from the entire coding sequence of the SYNGAP1 gene in the four clone hiPSCs. Red arrow indicates predicted Cas9 cut site. Numbers indicate clonal reads relative to Cas9 hiPSC reads. G, Indels from each clone identified from WES analysis. Indels were identified by clonal sequence differences from the original Cas9 hiPSCs (reference sequence). Indel threshold was determined by at least $50 \%$ of the reads differing from the reference sequence with a minimum of at least 10 reads. Indels with frequency $>0.8$ were used to determine frequency of homozygous variants. *(in clone \#4) indicates the compound heterozygous deletion of neighboring base pairs on each copy of SYNGAP1 within exon 7 ( $\boldsymbol{B}, \boldsymbol{C})$. ${ }^{*}$ (in clone \#38) indicates the 8 bp deletion present in one copy of SYNGAP1. The other copy contains a large deletion encompassing the entirety of exons 6 and 7 . $\boldsymbol{H}$, Whole-genome view of iNeurons from WT\#6, WT\#30, KO\#4, and KO\#38 clones represents a copy number value of 2 cross all chromosomes (except for the Y-chromosome, which is not detected), revealing normal (female) karyotype with no chromosomal aberrations. Pink, green, and yellow represent the raw signal for each individual chromosome probe. Blue signal represents the normalized probe signal that is used to identify copy number and aberrations (if any). measured at DIV45 by tracing dendrites of sparsely labeled eGFP-positive iNeurons (Fig. 3A). Relative to each isogenic control line, total dendritic fields were substantially larger in iNeurons derived from SYNGAP1$\mathrm{KO}$ clones. This difference was observed at the level of individual clones (Fig. $3 A, B$; $\mathrm{H}=54.81, p \leq 0.0001 ; N=30$ cells per clone) and when clones were grouped by genotype (Fig. $3 B ; U=436, p \leq 0.0001 ; N=60$ cells per genotype). Examination of the length by dendritic category (e.g., primary) revealed that, compared with WT clones, KO clones generally had longer primary and secondary dendrites (Fig. 3A,C-E; Primary dendrites: clonal analysis, $\mathrm{H}=49.71, p<0.0001, N=30$ cells per clone; genotype analysis, $U=545$, $p<0.0001, \quad N=60$ cells per genotype; Secondary dendrites: clonal analysis, $\mathrm{H}=$ 20.45, $p<0.0001 ; N=30$ cells for WT\#6 and KO\#38; $N=26$ cells for WT\#30; $N=27$ cells from $\mathrm{KO} 4$; genotype analysis, $U=880$, $p<0.0001 ; N=56$ cells from WT genotype; $N=57$ cells from KO genotype; Tertiary dendrites: clonal analysis, $\mathrm{H}=7.115, p=0.0683$; $N=6$ dendrites of 30 cells from WT\#6, $n=5$ dendrites of 30 cells from WT\#30, $n=4$ dendrites of 30 cells from $\mathrm{KO} 44$ and $n=4$ dendrites of 30 cells from $\mathrm{KO} 338$; genotype analysis, $U=73.55, p=0.0068 ; N=11$ cells from WT genotype; $N=8$ cells from $\mathrm{KO}$ genotype). The lack of a clonal difference within tertiary dendrites likely reflected a lower statistical power, as many neurons lacked these structures. In contrast to length, the complexity of dendritic arbors was unaffected by SYNGAP1 disruption. Clonal and genotype effects of SYNGAP1 were not observed for total dendrites (Fig. 3F; Total dendrites: clonal analysis, $\mathrm{H}=5.957, p=$ $0.1137 ; N=30$ cells per clone; genotype analysis, $U=1613, p=0.3222 ; N=60$ cells per genotype). Moreover, no SYNGAP1 effects were observed for each dendrite subtype (Fig. 3G-I; Primary dendrites: clonal analysis, $\mathrm{H}=1.680, p=0.6413, n=30$ cells per clone; genotype analysis, $U=1639, p=0.3755$, $n=60$ cells per genotype; Secondary dendrites: clonal analysis, $\mathrm{H}=10.72, p=0.0133$, $n=30$ cells per clone for clone comparisons; genotype analysis, $U=1689, p=0.5552$, $n=60$ cells per genotype; Tertiary dendrites: clonal analysis, $\mathrm{H}=0.4531, p=0.9291$, $n=30$; genotype analysis, $U=1731, p=$ $0.6129, n=60$ cells per genotype).

Tracing studies suggested that reduced SynGAP expression leads to iNeurons with larger dendritic fields. To confirm this, we performed an orthogonal analysis, consisting of immunocytochemical labeling of dendritic and synaptic proteins, in neurons derived from one pair of isogenic WT or KO iPSCs (Fig. $4 A, B$ ). The MAP2 area was enhanced in 
Table 1. Clonal variants identified through WES

\begin{tabular}{|c|c|c|c|c|c|c|}
\hline Alternative frequency & Chromosome & Position & Symbol & Reference allele & Alternative allele & Mutation \\
\hline \multicolumn{7}{|l|}{ WT\#6 } \\
\hline 0.5 & 10 & 19352491 & MALRD1 & AGT & A & Frameshift \\
\hline 0.5 & 17 & 80994507 & CHMP6 & $\begin{array}{l}\text { TGCCTGGCGCTCAGTAGCGTGGCCAG/ } \\
\text { GGCTCCCAGTGTGGGCTCGGTGAC }\end{array}$ & $\mathrm{T}$ & Frameshift \\
\hline 0.5 & 20 & 24930694 & & TC & $\mathrm{T}$ & \\
\hline 0.529 & 5 & 176508392 & FAF2 & $C$ & CA & Frameshift \\
\hline 0.554 & 8 & 58426202 & UBXN2B & CT & $c$ & Frameshift \\
\hline \multicolumn{7}{|l|}{ WT\#30 } \\
\hline 0.5 & 21 & 10483254 & BAGE2 & $\mathrm{T}$ & TA & Frameshift \\
\hline 0.5 & 8 & 8066187 & & $\mathrm{~T}$ & TG & \\
\hline 0.502 & 14 & 69119490 & DCAF5 & $\mathrm{T}$ & TA & Frameshift \\
\hline 0.502 & 19 & 54456134 & LENG8 & TCAC & $T$ & Deletion \\
\hline 0.507 & 6 & 4121693 & $\mathrm{ECI} 2$ & $G$ & GT & Frameshift \\
\hline 0.643 & 9 & 91711733 & ROR2 & G & GGTA & Insertion \\
\hline \multicolumn{7}{|l|}{ KO\#4 } \\
\hline 0.5 & 9 & 78300710 & PSAT1 & $C$ & CTT & Frameshift \\
\hline 0.5 & 9 & 91711722 & ROR2 & TGTAA & $\mathrm{T}$ & Frameshift \\
\hline 0.503 & 13 & 108228871 & ABHD13 & TA & $\mathrm{T}$ & Frameshift \\
\hline 0.505 & 18 & 23795721 & LAMA3 & TA & $\mathrm{T}$ & Frameshift \\
\hline 0.553 & 22 & 31711776 & PRR14L & $C$ & $\mathrm{CA}$ & Frameshift \\
\hline 0.556 & 21 & 43757986 & PDXK & CT & $C$ & Frameshift \\
\hline 0.6 & $x$ & 12817468 & PRPS2 & $T$ & TA & Frameshift \\
\hline 0.614 & 17 & 3814729 & NCBP3 & $C$ & CT & Frameshift \\
\hline 0.616 & 19 & 41837694 & LYPD4 & $C$ & CA & Frameshift \\
\hline 0.616 & 1 & 6581663 & ZBTB48 & $C A$ & $C$ & Frameshift \\
\hline 0.545 & 16 & 11156369 & CLEC16A & $C$ & CA & Frameshift \\
\hline 0.554 & 18 & 59824581 & & A & AT & \\
\hline 0.573 & 19 & 17988154 & KCNN1 & $C$ & CA & Frameshift \\
\hline 0.578 & 5 & 138505807 & & $\mathrm{~T}$ & TA & \\
\hline 0.584 & 1 & 27552400 & AHDC1 & CT & $C$ & Frameshift \\
\hline 0.585 & 6 & 34219990 & & C & CAAA & \\
\hline 0.616 & 7 & 101627137 & MYL10 & $C$ & CAAAAA & Frameshift \\
\hline 0.666 & 7 & 48844654 & & A & AT & \\
\hline 0.778 & 9 & 130349951 & HMCN2 & G & GCAGTGTT & Frameshift \\
\hline 0.933 & 6 & 33435544 & SYNGAP1 & TGCCTGTCG & $\mathrm{T}^{b}$ & Frameshift \\
\hline
\end{tabular}

${ }^{a}$ Compound heterozygosity; combined reads for the both SYNGAP1 copies.

${ }^{b}$ Only one SYNGAP1 copy in this region was read-mapped by WES; the other copy contained a large deletion in this region.

KO cultures (Fig. 4C; MAP2 comparison: $U=2181, p<0.0001$, $N=85$ images for WT\#30 and $N=81$ images for KO\#4). This was not because of a higher density of $\mathrm{KO}$ iNeurons compared with WT controls (Fig. 4D; number of soma comparison: $U=2746, p=0.0240, N=85$ for WT\#30 and $N=81$ for $\mathrm{KO} \# 4)$. Indeed, a large effect size for MAP2 area persisted when this measure was normalized to soma count (Fig. 4E; MAP2 area/ soma count comparison: $U=2097, p<0.0001 N=85$ for WT\#30

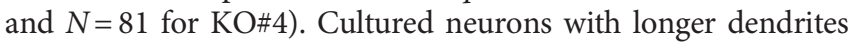
would be expected to have an increase in absolute numbers of postsynaptic structures. Indeed, absolute numbers of PSD95 and GLUA1 structures were also increased in the KO culture
(Fig. 4C; PSD95: $U=1891, p<0.0001, N=85$ for WT\#30 and $N=81$ for KO\#4; GluA1: $U=1999, p<0.0001 N=85$ for WT\#30 and $N=81$ for KO\#4). The effect of genotype on synaptic labeling was still significant, albeit with a much smaller effect size, when PSD95 and GLUA1 structures were normalized to MAP2 area (Fig. 4F; PSD95 objects/MAP2 area comparison: $U=2752, p=0.0255, N=85$ for WT\#30 and $N=81$ for KO\#4; GluA1 objects/MAP2 area comparison: $t_{(164)}=$ 2666, $p=0.0084, N=85$ for WT\#30 and $N=81$ for KO\#4). These labeling studies support the idea that disrupting SynGAP expression results in cultures comprised of larger neurons with more postsynaptic structures. 
A

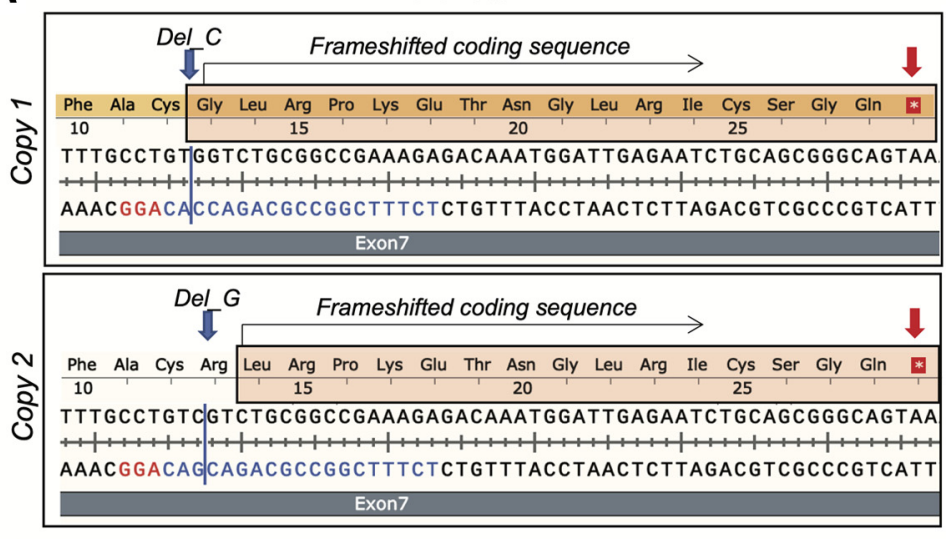

B
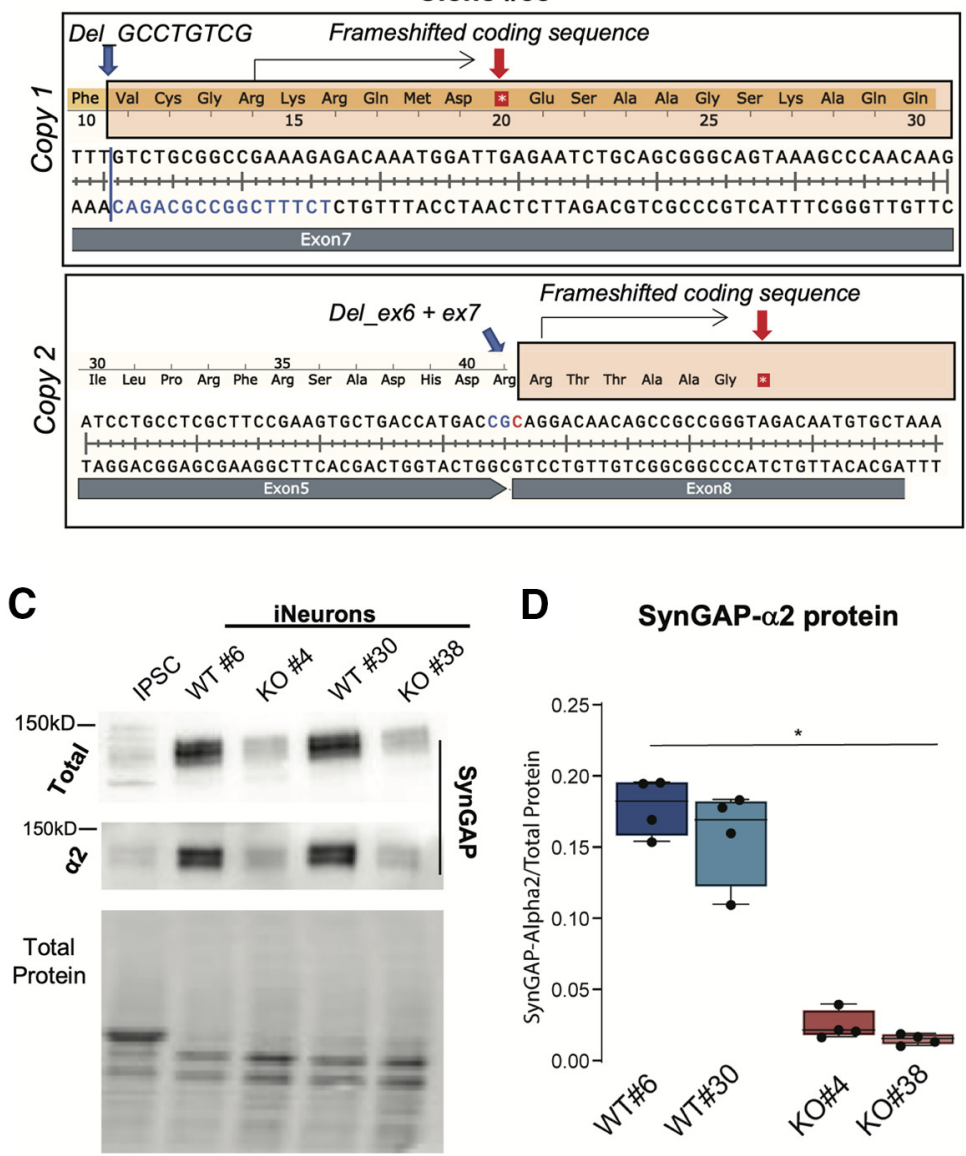

D SynGAP- $\alpha 2$ protein

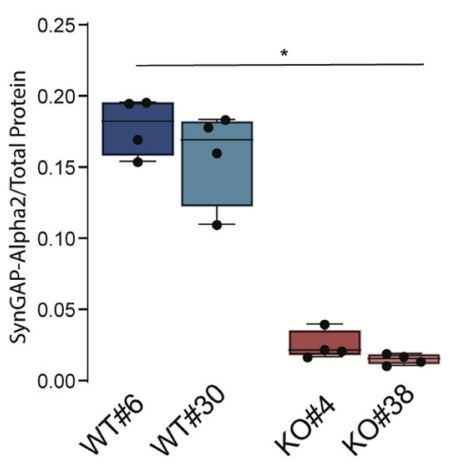

Figure 2. Nominal SynGAP protein expression in clones \#4 and \#38. $A, B$, Model, based on evidence from Sanger traces and WES, of how targeted CRISPR mutations impacted each copy of SYNGAP1 in the two "KO" clones. Predicted impact on coding sequences is also included. C, Western blots demonstrating SynGAP protein expression from iNeuron or hiPSC homogenate. Total refers to signal from an antibody that detects all splice variants, and $\alpha 2$ refers to signal from an antibody that detects only a specific C-terminal splice variant. $\boldsymbol{D}$, Quantification of relative intensity of bands normalized to total protein signal. In box-and-whisker plots, the middle, boxes, and whiskers represent the median, interquartile range, and min to max, respectively. ${ }^{*} p<0.05$.

The observation of larger iNeurons with increased numbers of postsynaptic structures prompted us to investigate the functional maturation of iNeurons with reduced SynGAP protein expression. Intrinsic membrane properties and the onset of glutamatergic synaptic activity are two measures that are developmentally regulated in Ngn2-iNeurons (Zhang et al., 2013). To test the idea that reducing SynGAP expression alters the maturation of iNeurons, we performed whole-cell voltage-clamp recordings at two developmental time points (DIV20-DIV30 and DIV40-DIV50; Fig. 5A,B). At DIV20DIV30, intrinsic membrane properties of all clones were characteristic of immature neurons (i.e., relatively low capacitance and high input resistance; Fig. 5C,D). We did not observe clonal or genotype differences in resting membrane potential, capacitance, or resistance at this time point (Fig. $5 C-E$; Membrane potential: clonal analysis, $F_{(3.95)}=$ $0.5132, p=0.6742, n=29$ cells from WT\#6, 31 cells from $\mathrm{WT} \# 30$, 34 cells from $\mathrm{KO} \# 4$, and 21 cells from KO\#38; genotype analysis, $t_{(97)}=0.08684, p=0.9310, n=51$ cells for $\mathrm{WT} \# 6+\mathrm{WT} \# 30$ and 48 cells for KO\#4+ KO\#38; Capacitance: clonal analysis, $\mathrm{H}=$ 3.123, $p=0.3730, n=29$ cells from WT\#6, 31

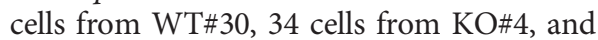
21 cells from KO\#38; genotype analysis, $U=1584, \quad p=0.5093 ; \quad N=62$ cells from $\mathrm{WT} \# 6+\mathrm{WT} \# 30$ and 55 cells from KO\#4+ KO\#38; Membrane resistance: clonal analysis, $\mathrm{H}=4.259, \quad p=0.2348, \quad n=28$ cells from $\mathrm{WT} \# 6,31$ cells from WT\#30, 34 cells from $\mathrm{KO} 4$, and 21 cells from KO\#38; genotype analysis, $U=1546, p=0.5619 ; N=60$ cells from $\mathrm{WT} \# 6+\mathrm{WT} \# 30$ and 55 cells from KO\#4 + KO\#38). However, we did observe that neurons made from SYNGAP1-KO hiPSCs showed earlier synaptic activity during development. Although some iNeurons from all clones exhibited mEPSCs at this time point (Fig. 5F), the proportion of mEPSC-expressing iNeurons was significantly increased in $\mathrm{KO}$ clones (Fig. 5G; $p=0.0008$ for $\mathrm{KO} \# 4$ vs WT $\# 6 ; p=0.0002$ for $\mathrm{KO} \# 38$ vs WT\#6, $p=0.0644$ for KO\#4 vs $\mathrm{WT} \# 30, n=20$ for KO\#4 and 18 for WT\#30, respectively; $p=0.0231$ for $\mathrm{KO} \# 38$ vs WT\#30; $N=12$ cells from WT\#6, 18 cells from WT\#30, 20 cells from KO\#4, and 19 cells from KO\#38). When grouping iNeurons by genotype, $\mathrm{KO}$ neurons were almost twice as likely to express miniature events (Fig. 5G; $p=0.0042$ for $\mathrm{KO} \# 4+\mathrm{KO} \# 38$ vs $\mathrm{WT} \# 6+$ $\mathrm{WT} \# 30, n=30$ cells from WT\#6 + WT\#30 and 39 cells from $\mathrm{KO} \# 4+\mathrm{KO} \# 38$ ). mEPSC frequency was low and variable at this early time point, making it difficult to compare clones or even genotypes (Fig. 5H,I; clonal analysis, $\mathrm{H}=1.910, p=0.5912, n=12$ cells from WT\#6, 18 cells from WT\#30, 20 cells from KO\#4, and 19 cells from KO\#38; genotype analysis, $U=504.5, p=0.5607 ; N=29$ cells from WT\#6+WT\#30 and 38 cells from KO\#4 + KO\#38, K-S test, $\mathrm{D}=0.2660, p<0.0001, n=951$ events from 29 cells from WT\#6+WT\#30, $n=1559$ events from 38 cells from KO\#4+KO\#38). In contrast, mEPSC amplitude was less variable. There appeared to be a weak clonal and genotype effect on mEPSC amplitude. Both KO amplitude populations exhibited a rightward shift compared with the two WT 

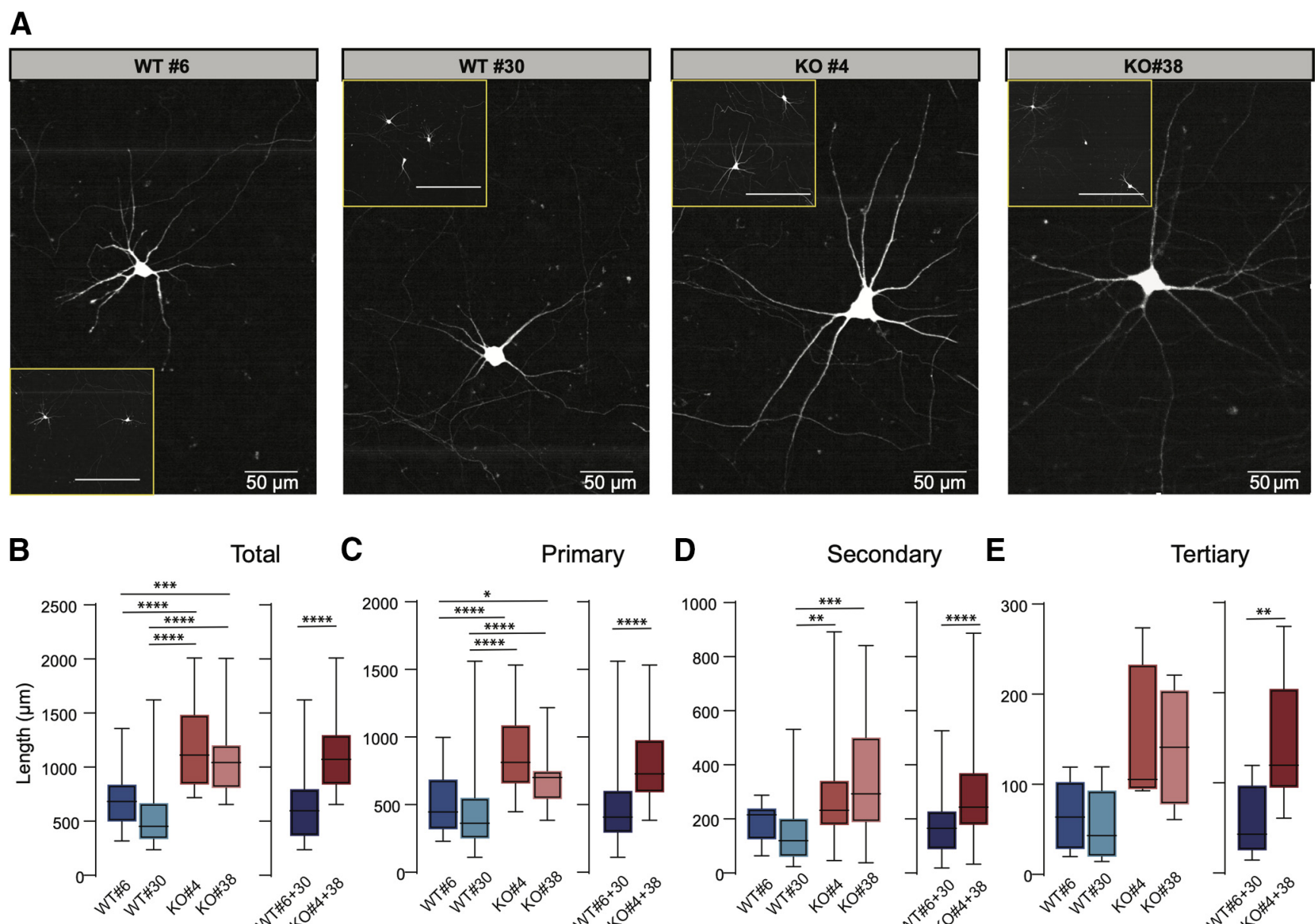

Total C

Primary

D

Secondary

E

Tertiary

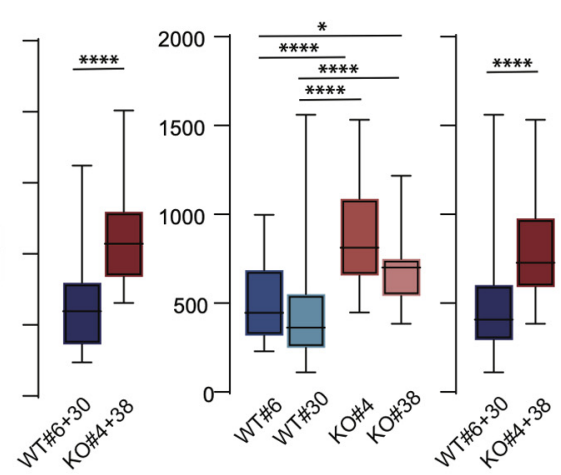

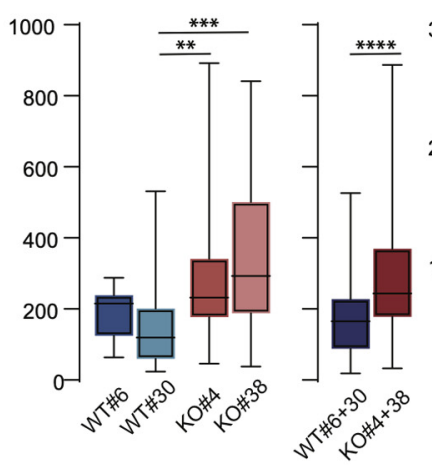

H

Secondary

I

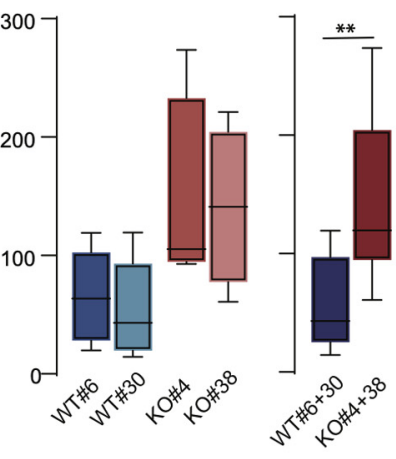

G

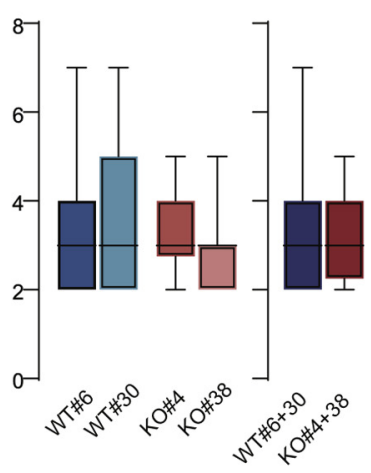

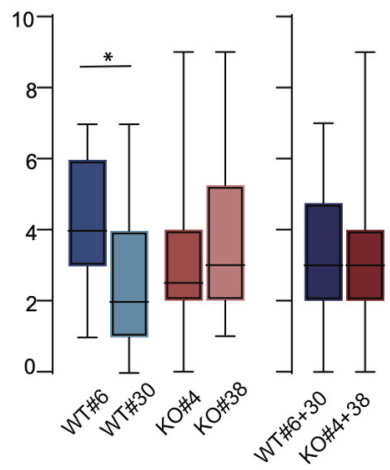

Tertiary

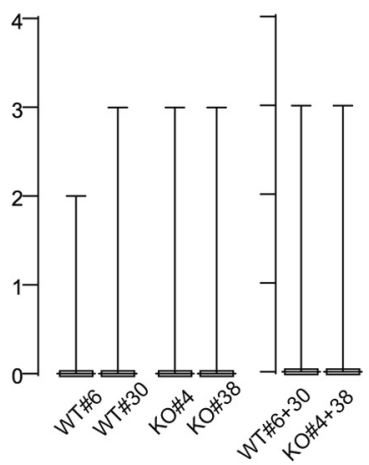

Figure 3. Increased dendrite length in iNeurons derived from KO iPSC clones. $A$, Representative images of eGFP-expressing iNeurons from the four different clones at DIV45. Scale bars: Inset, $200 \mu \mathrm{m} . \boldsymbol{B}-\boldsymbol{E}$, Histograms represent average length per cell of total $(\boldsymbol{B})$, primary $(\boldsymbol{C}$, secondary $(\boldsymbol{D})$, and tertiary dendrites $(\boldsymbol{E})$ of the four clones. $\boldsymbol{F}-\boldsymbol{I}$, Graphs represent average number of dendrites per cell of total $(\boldsymbol{F})$, primary $(\boldsymbol{G})$, secondary $(\boldsymbol{H})$, and tertiary $(\boldsymbol{I})$ dendrites of the four clones. In box-and-whisker plots, the middle, boxes, and whiskers represent the median, interquartile range, and min to max, respectively. ${ }^{*} p<0.05,{ }^{* *} p<0.01,{ }^{* * *} p<0.001,{ }^{* * *} p<0.0001$.

populations (Fig. 5J; $\mathrm{H}=7.565, p=0.0559, n=15$ cells from WT\#6, 20 cells from WT\#30, 19 cells from KO\#4, and 19 cells from $\mathrm{KO} \# 38$ ). When clonal data were collapsed by genotype, a robust statistical effect emerged at the level of individual events and at the level of cellular population means (Fig. 5J; $U=504.5$, $p=0.5607 ; N=29$ cells from WT\#6 $+\mathrm{WT} \# 30$ and 38 cells from $\mathrm{KO} \# 4+\mathrm{KO} \# 38$, K-S test, $\mathrm{D}=0.2660, p<0.0001, n=981$ events from 35 cells from WT\#6 + WT\#30, $n=1601$ events from 38 cells from $\mathrm{KO} 4+\mathrm{KO} 38$ ).

We next analyzed synaptic activity in more mature iNeurons (DIV40-DIV50; Fig. 5L). As a population, neurons derived from
WT clones were roughly twice as likely to express synaptic activity at this time point compared with younger neurons of the same genotype, indicative of substantial neuronal maturation during this period (Fig. 5G,M). However, this effect was less pronounced in $\mathrm{KO}$ neurons (Fig. $5 G, M$ ). There was a significant effect of time on the proportion of neurons expressing synaptic activity in WT neurons, but this effect was absent in $\mathrm{KO}$ iNeurons $(p=0.0004$ for WT\#6 +WT\#30, DIV40-DIV50 vs DIV20-DIV30; $p=0.0592$ for KO\#4 + KO\#38, DIV40-DIV50 vs DIV20-DIV30). There was no longer an effect of genotype on the proportion of neurons with synaptic activity at the more 
A
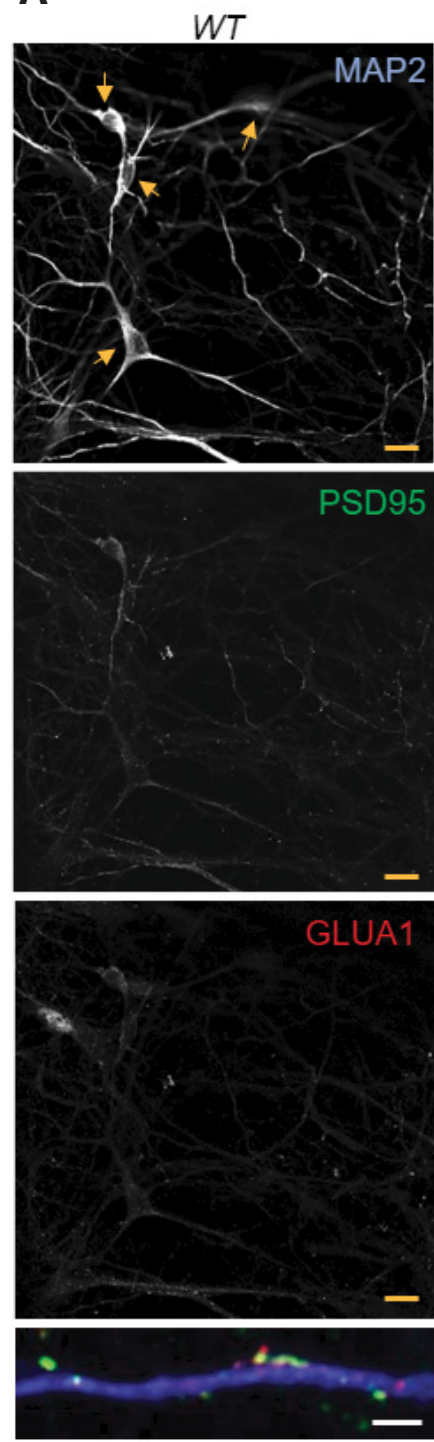

B
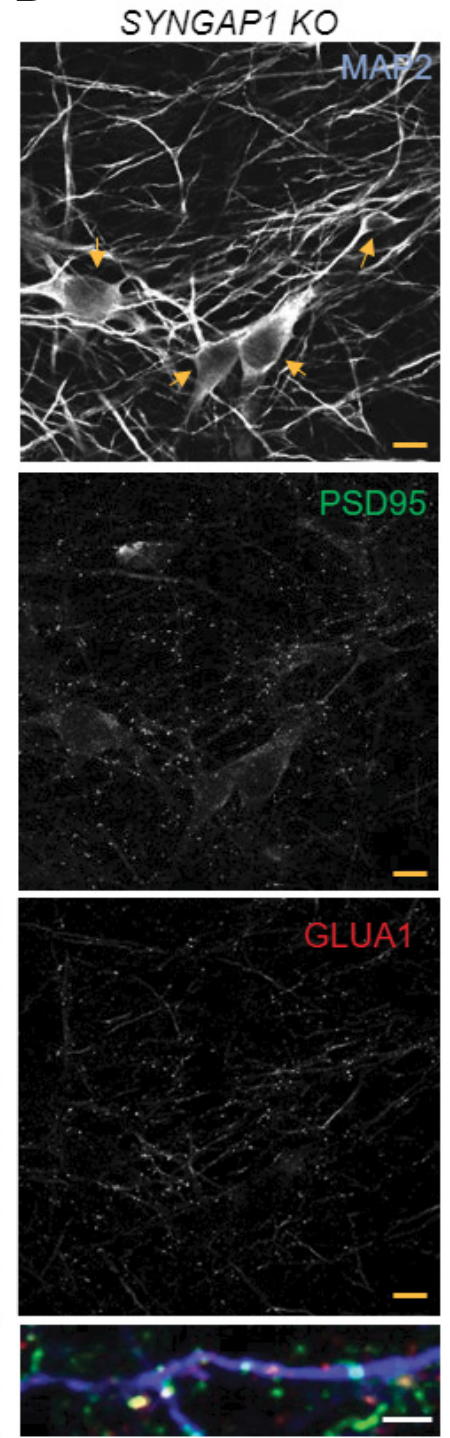

C
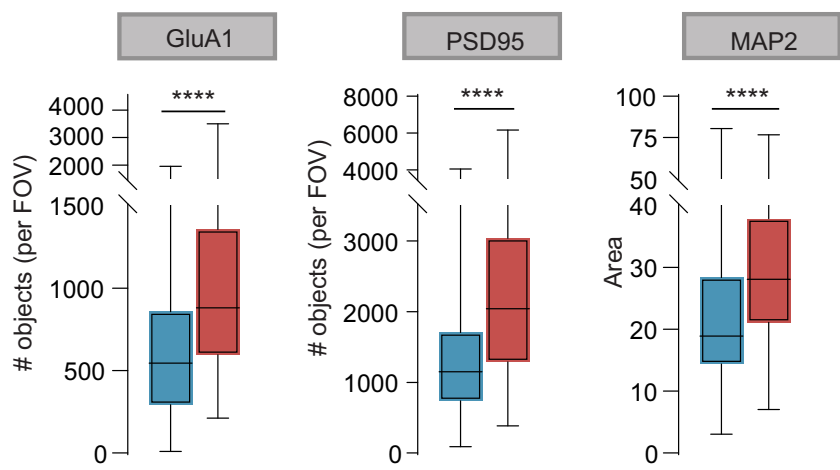

D

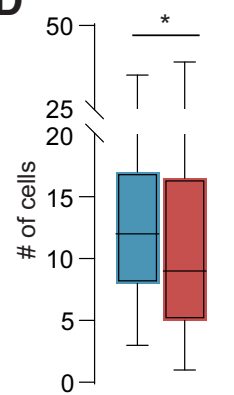

E

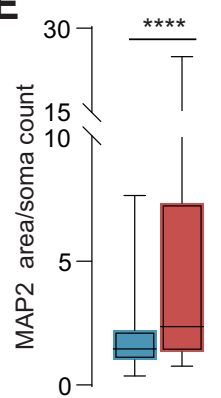

F
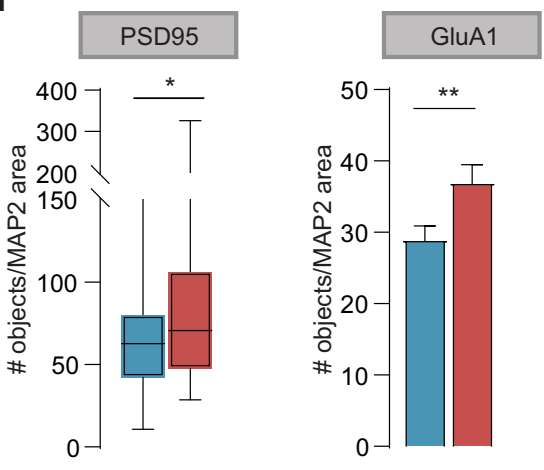

WT\#30

Figure 4. Increased dendritic area and more numerous postsynaptic structures in SYNGAP1 KO iNeurons. $\boldsymbol{A}, \boldsymbol{B}$, Representative images showing MAP2 labeling (top), PSD95 labeling (middle), and GluA1 labeling (middle bottom), and merge of MAP2, PSD95, and GLUA1 (bottom) of iNeurons from WT\#30 ( $\boldsymbol{A})$ and KO\#4 (B) at DIV45. Yellow arrows indicate cell bodies. Scale bars: yellow, $10 \mu \mathrm{m}$; white, $2 \mu \mathrm{m}$. C, Graphs represent MAP2 area and number of PSD95 and GluA1 objects (punctate labeling) per FOV in WT\#30 and KO\#4. D, Number of MAP2-postive somas detected per image FOV. $\boldsymbol{E}$, MAP2 signal normalized to MAP2 = positive somas per image FOV. $\boldsymbol{F}$, Graphs represent quantification of PSD95 and GluA1 expression in WT\#30 and KO\#4 normalized to MAP2 area. In box-and-whisker plots, the middle, boxes, and whiskers represent the median, interquartile range, and min to max, respectively. Bar graph represents mean \pm SEM. ${ }^{*} p<0.05,{ }^{* *} p<0.01,{ }^{* * * *} p<0.0001$.

mature stage of development ( $p=0.2399$ for WT\#6 $+\mathrm{WT} \# 30$ vs $\mathrm{KO} \# 4+\mathrm{KO} \# 38 ; n=50$ cells from WT\#6 $+\mathrm{WT} \# 30$ and 43 cells from $\mathrm{KO} 44+\mathrm{KO} 38$ ). Within the population of neurons with synaptic events, we measured mEPSC frequency and amplitude. The frequency of events was highly variable in these populations (Fig. 5N,O), which made it difficult to draw clear conclusions across clones and genotypes. There was a trend toward more frequent events in combined KO populations, although these trends were not apparent when looking at individual clones (clonal analysis, $\mathrm{H}=2.874, p=0.4115, n=21$ cells from WT\#6, 28 cells from WT\#30, 24 cells from KO\#4, and 18 cells from KO\#38; genotype analysis, $U=970.5, \quad p=0.644 ; n=49$ cells from $\mathrm{WT} \# 6+\mathrm{WT} \# 30$, and $n=42$ cells from KO\#4+KO\#38, K-S test, $\mathrm{D}=0.2763, p<0.0001, n=2182$ events from 49 cells from WT\#6 + WT\#30, $n=2498$ events from 42 cells from KO\#4+ $\mathrm{KO \# 38)}$. With respect to amplitude (Fig. 5P,Q), we once again observed a weak effect of clone and genotype at this time point that was consistent with observations from developmentally younger iNeurons. Neurons from both KO clones appeared to have slightly larger events compared with those from WT iNeurons. This effect was apparent in comparisons of mEPSC distributions of all events (Fig. 5P,Q; K-S test, $\mathrm{D}=0.2990, p<0.0001, n=2254$ events from 49 cells from $\mathrm{WT} \# 6+\mathrm{WT} \# 30, n=2554$ events from 42 cells from $\mathrm{KO} 44+$

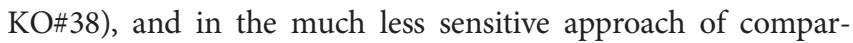
ing event means from individual neurons (Fig. 5P,Q, inset; clonal analysis, $F_{(3.87)}=3.73, p=0.0142, p=0.0187$ for $\mathrm{KO} 4$ vs WT\#6, $p=0.0499$ for $\mathrm{KO \# 4}$ vs WT\#30, $p=0.9407$ for $\mathrm{WT} \# 6$ vs WT\#30, $p=0.3151$ for WT\#6 vs KO\#38, $p=0.5696$

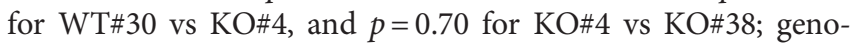
type analysis, $t_{(89)}=3.121, p=0.0024, n=49$ cells for WT\#6 + $\mathrm{WT} \# 30$ and 42 for $\mathrm{KO} 44+\mathrm{KO} \# 38)$. 
The effect of SynGAP expression on iNeuron mEPSC frequency and amplitude was somewhat consistent across developmental time points, but the effect sizes, when present, were relatively small. To determine whether these effects were reproducible, we performed an additional experiment on iNeurons produced from the same clones. Data for this experiment were collected from a completely new hiPSC expansion and neuronal induction procedure. In this additional experiment, we observed similar effects of SynGAP expression on intrinsic membrane properties and mEPSCs (Fig. 6A-G). SYNGAP1 deletion did not affect the resting membrane potential, input resistance, or capacitance at the clonal or genotype level (Fig. 6A-C; Membrane potential: clonal analysis, $F_{(3.54)}=0.5456, p=0.6532, n=11$ cells from WT\#6, 16 cells from WT\#30, 13 cells from $\mathrm{KO \# 4}$, and 18 cells from $\mathrm{KO \# 38;} \mathrm{genotype} \mathrm{analysis,} t_{(56)}=1.215$, $p=0.2295, n=27$ cells for WT\#6 + $\mathrm{WT} \# 30$ and 21 for $\mathrm{KO} 44+\mathrm{KO} 338$; Capacitance: clonal analysis, $\mathrm{H}=9.091, p$ $=0.0281, p=0.0318$ for WT\#6 vs $\mathrm{WT} \# 30, n=28$ cells from WT\#6, 41 cells from WT\#30, 34 cells from $\mathrm{KO} 4$, and 50 cells from $\mathrm{KO} \# 38$; genotype analysis, $U=2828, p=0.7973 ; N=69$ cells from $\mathrm{WT} \# 6+\mathrm{WT} \# 30$ and 84 cells from $\mathrm{KO} 4+\mathrm{KO} 38$; Membrane resistance: clonal analysis, $\mathrm{H}=4.738, \quad p=0.1920$, $n=28$ cells from WT\#6, 41 cells from $\mathrm{WT} \# 30,34$ cells from $\mathrm{KO \# 4}$, and 50 cells from $\mathrm{KO} \# 38$; genotype analysis, $U=2896, p=0.9949 ; N=69$ cells from $\mathrm{WT} \# 6+\mathrm{WT} \# 30$ and 84 cells from

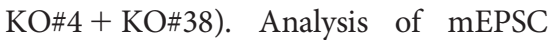
frequency from each of the clones revealed a trend for increased frequency from neurons with disruptive SYNGAP1 variants (Fig. 6D). The two $\mathrm{KO}$ clones have a greater frequency of mEPSCs when looking at cumulative probability distributions, and this drove an effect at the genotype level (Fig. 6E; K-S test, $\mathrm{D}=0.1744, p<0.0001, n=1199$ events from 20 cells from WT\#6 + WT\#30, $n$ $=1512$ events from 21 cells from $\mathrm{KO} 4+\mathrm{KO}$ 38). A statistical effect was not present when comparing cellular means of mEPSC frequency (clonal analysis, $\mathrm{H}=1.663$, $p=0.6452, n=11$ cells from WT\#6, 9 cells from WT\#30, 8 cells from $\mathrm{KO} 44$, and 13 cells from $\mathrm{KO} 38$; genotype analysis, $U=164, p=0.2382 ; N=20$ cells from WT\#6 + WT\#30 and

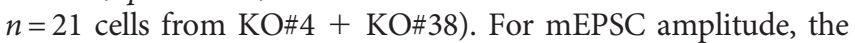
clonal and genotype effects were clearer compared with frequency measures. The cumulative distribution for mEPSC amplitudes for all events clearly shifted to larger values in both $\mathrm{KO}$ clones (Fig. 6F). This drove a substantial and highly significant

A
B
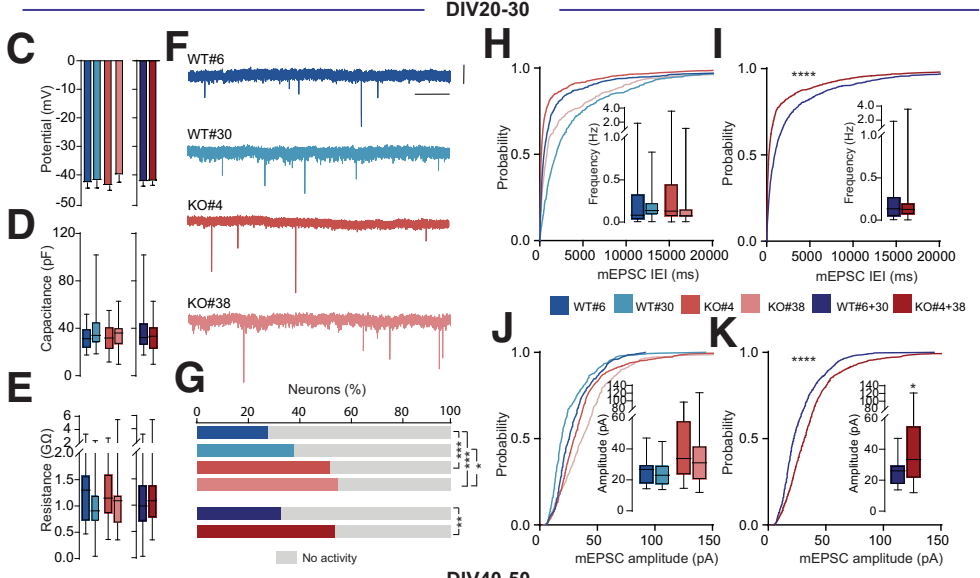

N
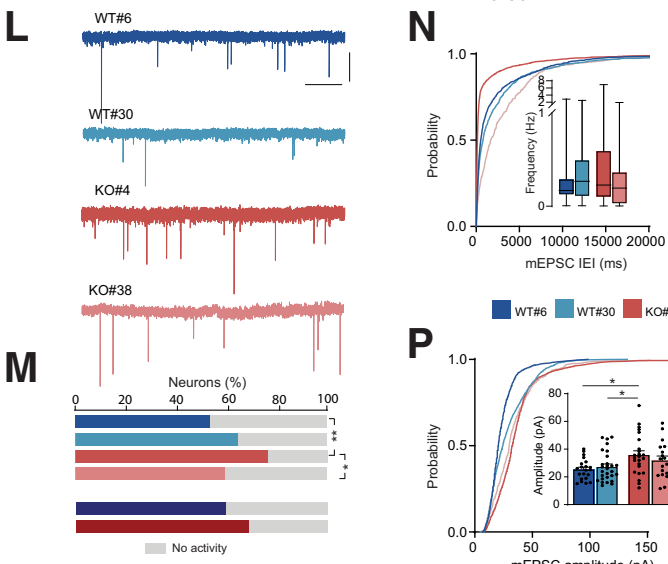

0

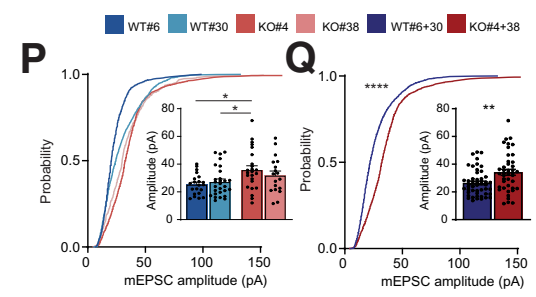

Figure 5. SYNGAP1 expression in human iNeurons regulates excitatory synapse function. $\boldsymbol{A}$, Flow diagram of iNeuron generation from WT and SYNGAP1 KO iPSCs for whole-cell electrophysiological experiments (recording days within red boxes). $\boldsymbol{B}$, Representative DIC image of patched iNeurons cells from WT\#6. C-E, Bar graphs represent intrinsic membrane properties measured at DIV20-DIV30 as resting membrane potential $(\boldsymbol{C}$, capacitance $(\boldsymbol{D})$, and input resistance $(\boldsymbol{E})$ from the four clones. $\boldsymbol{F}$, Representative traces of mEPSCs of iNeurons from WT and K0 clones at DIV20-DIV30. Calibration: 2 s, $20 \mathrm{pA}$. G, Percentage of successful observations of mEPSCs in iNeurons from the four clones at DIV20-DIV30. $\boldsymbol{H}, \mathbf{I}$, Cumulative plots of mEPSC interevent interval and frequency (inset) of the different clones individually $(\boldsymbol{H})$ and grouped by genotype $(\boldsymbol{I})$ at DIV20-DIV30. $J, K$, Cumulative probability plots of mEPSC amplitude of the different clones individually $(\boldsymbol{J})$ and grouped by genotype $(K)$ at DIV20-DIV30. $\boldsymbol{L}$, Representative traces of mEPSCS of iNeurons from WT and K0 clones at DIV40-DIV50. Calibration: $2 \mathrm{~s}, 20 \mathrm{pA}$. $\boldsymbol{M}$, Percentage of successful observations of mEPSCs in iNeurons from the four clones at DIV40-DIV50. $\boldsymbol{N}, \mathbf{O}$, Cumulative probability plots of mEPSC interevent interval (IEI) and frequency (inset) of the different clones individually $(\boldsymbol{N})$ and grouped by genotype $(\boldsymbol{O})$ at DIV40-DIV50. $\boldsymbol{P}, \mathbf{Q}$, Cumulative probability plots of mEPSC amplitude of the different clones individually $(\boldsymbol{P})$ and grouped by genotype $(\boldsymbol{Q})$ at DIV40-DIV50. In box-and-whisker plots, the middle, boxes, and whiskers represent the median, interquartile range, and min to max, respectively. Bar graphs represent mean \pm SEM. ${ }^{*} p<0.05,{ }^{* *} p<0.01,{ }^{* * *} p<0.001,{ }^{* * * *} p<0.0001$.

shift in the disruption at the genotype level (Fig. 6G; K-S test, $\mathrm{D}=0.2954, p<0.0001, n=1085$ events from 20 cells from WT\#6 $+\mathrm{WT} \# 30, n=1396$ events from 21 cells from KO\#4 + KO\#38). We did not observe an effect on population means when looking at cellular averages (clonal analysis, $\mathrm{H}=7.565, p=0.0559, n=15$ cells from WT\#6, 20 cells from WT\#30, 19 cells from KO\#4, and 19 cells from KO\#38; genotype analysis, $U=504.5, p=0.5607$; $N=29$ cells from WT\#6 $+\mathrm{WT} \# 30$ and 38 cells from $\mathrm{KO} 44+$ $\mathrm{KO \# 38).} \mathrm{However,} \mathrm{the} \mathrm{power} \mathrm{for} \mathrm{this} \mathrm{experiment} \mathrm{was} \mathrm{lower} \mathrm{than}$ the one presented in Figure 5. Together, we conclude that 


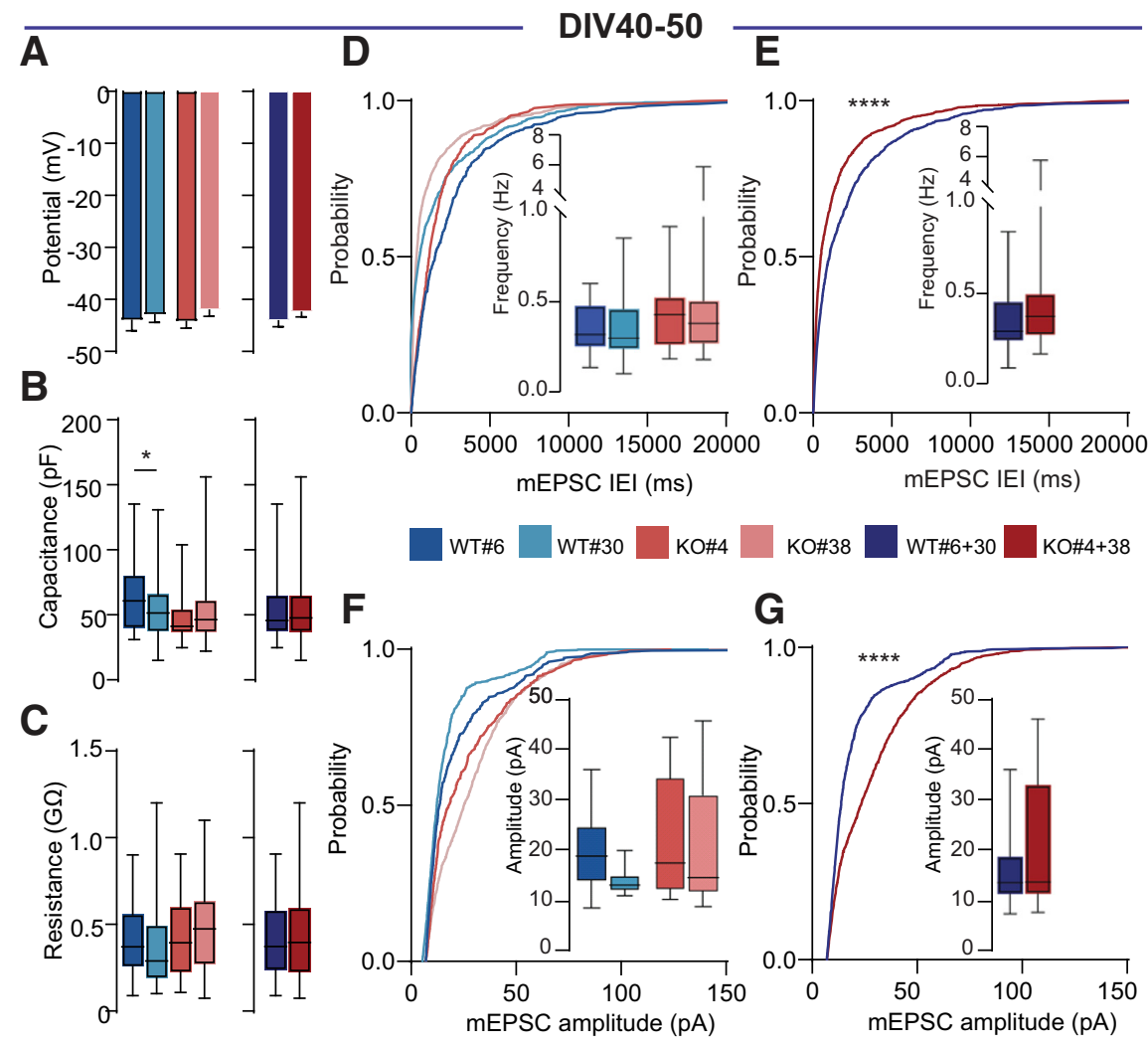

Figure 6. Reproducibility of SYNGAP1-mediated effects on iNeuron excitatory synapse function. $\boldsymbol{A}-\boldsymbol{C}$, Graphs represent resting membrane potential $(\boldsymbol{A})$, capacitance $(\boldsymbol{B})$, and input resistance $(\boldsymbol{C})$ from the four clones at DIV40-DIV50. D, $\boldsymbol{E}$, Cumulative plots of mEPSC interevent interval (IEI) and frequency (inset) of the different clones individually $(\boldsymbol{D})$ and grouped by genotype $(\boldsymbol{E})$ at DIV40-DIV50. $\boldsymbol{F}, \boldsymbol{G}$, Cumulative probability plots of mEPSC amplitude of the different clones individually $(\boldsymbol{F})$ and grouped by genotype $(\boldsymbol{G})$ at DIV40-DIV50. In box-and-whisker plots, the middle, boxes, and whiskers represent the median, interquartile range, and min to max, respectively. Bar graphs represent mean \pm SEM. ${ }^{*} p<0.05$, ${ }^{* * *} p<0.0001$.

reducing SynGAP expression in Ngn2 iNeurons leads to weak, but reproducible, effects on mEPSC amplitude. Effects on frequency were unclear because of high variability.

Our data demonstrate that reducing SynGAP expression results in larger iNeurons that exhibit early synaptic maturity. Therefore, we hypothesized that reducing SynGAP expression would also influence the development of network activity in cultured iNeurons. To test this, we measured spontaneous distributed network activity in cultures derived from KO and WT clones using an MEA system (Fig. 7A-C). For these studies, we derived cultures using the dual SMAD method (Jiang et al., 2017). This approach is advantageous for analysis of network activity because multiple brain cell types, including interneurons and glia, develop from this method. Therefore, developing networks make use of naturally occurring elements normally present in brain circuits. Recordings of the same cultures were performed over the course of several weeks, which enabled in vitro measurements of network spiking activity during neuronal development. From as early as week 2, we observed evidence of spiking activity in cultures derived from each of the iPSC clones. However, both SYNGAP1 KO clones exhibited substantially increased firing rates compared with isogenic controls (clone: $F_{(3,12)}=52.42, p<0.0001, N=4$ replicas for WT\#6, WT\#30, $\mathrm{KO \# 4}$, and $\mathrm{KO} \# 38$ ). The enhanced firing rate in KO iNeurons emerged progressively and was sustained through week 6 in culture at both clonal (Fig. $7 D$; time: $F_{(1.609,19.31)}=10.61, p=0.0014$; time $\times$ clone: $F_{(12,48)}=4.286, p=0.0001, N=4$ replicas for WT\#6, WT\#30, KO\#4, and KO\#38) and genotype levels (Fig. 7E; time: $F_{(2.029,28.41)}=8.739, p=0.0002$; time $\times$ genotype: $F_{(4,56)}=6.465, p<$ $0.0002, N=8$ replicas from WT\#6 + $\mathrm{WT} \# 30$ and $\mathrm{KO} \# 4+\mathrm{KO} \# 38)$. Next, we measured bursting activity in each of the four clones. We observed significantly elevated neuronal bursts in $\mathrm{KO}$ versus control neurons (Fig. 7F,G; clone: $F_{(3,12)}=21.97, p<0.0001, N=4$ replicas for $\mathrm{WT} \# 6$, $\mathrm{WT} \# 30, \mathrm{KO} \# 4$, and KO\#38). Quantification of distributed network connectivity demonstrated that $\mathrm{KO}$ neuronal cultures displayed different degrees of neural network activity, observed as "network bursts," as early as 3 weeks of maturation. Enhanced network bursting activity in KO cultures relative to WT controls was observed at both the clonal (Fig. $7 H$; clone: $F_{(3,12)}=15.23, p=0.0002$, $N=4$ replicas for WT\#6, WT\#30, $\mathrm{KO} 4$, and $\mathrm{KO} \# 38$ ) and genotype levels (Fig. 7I; genotype: $F_{(1,14)}=11.95$, $p=0.0039, N=8$ replicas from WT\#6 $+\mathrm{WT} \# 30$ and $\mathrm{KO} 4+\mathrm{KO} \# 38$ ). Thus, SYNGAP1 expression substantially influences the dynamics of cellular activity in developing neuronal networks.

\section{Discussion}

We produced iNeurons from human hiPSCs with a disrupted SYNGAP1 gene in an effort to understand how this gene shapes human neuron development and function. This is an important research question given that pathogenic SYNGAP1 variants cause a complex neurodevelopmental disorder defined by early-onset epilepsy, cognitive impairment, and autistic features (Hamdan et al., 2011; Jimenez-Gomez et al., 2019; Vlaskamp et al., 2019; Satterstrom et al., 2020). We found that SYNGAP1 regulates the postmitotic maturation of dendrites and synapses from human iNeurons. Cas9-mediated disruption of SYNGAP1 expression enhanced dendritic morphogenesis, accelerated the acquisition of synaptic activity, and drove increased spiking activity measured in functionally connected two-dimensional iNeuron cultures. Our data indicate that loss of SynGAP protein expression was responsible for the dendrite and synapse maturation phenotypes observed in these cultures. Indeed, we observed consistent structural phenotypes at the level of individual clones that were subsequently grouped by genotype. WES demonstrated that the only shared variants between the two KO clones were frameshift deletions in the SYNGAP1 gene, and immunoblotting confirmed that iNeurons derived from KO clones expressed nominal levels of SynGAP protein. Altered dendritic maturation was supported by data obtained from orthogonal experimental measures. We observed longer dendrites in eGFPpositive iNeurons, and an increased dendritic area measured from endogenous MAP2 signal. iNeurons derived from the KO hiPSC clone also exhibited an increase in the absolute density of postsynaptic structures, a finding consistent with a neuronal culture populated with neurons containing longer dendrites. Given that the length of dendrites and the density of postsynaptic 
structures in iNeurons increase over time in culture (Zhang et al., 2013), these data support the conclusion that SynGAP expression regulates the maturation rate of dendritic and synaptic structures in human iNeurons. This conclusion was also supported by clonal and genotype differences in synaptic activity between WT and KO iNeurons. Individual iNeurons have been shown to gradually acquire synaptic activity in the first several weeks in culture (Zhang et al., 2013; Nehme et al., 2018). However, we found that KO neurons expressed synaptic activity earlier in development compared with WT neurons.

Distributed neuronal activity, measured by MEA analysis, confirmed that structural maturation of dendrites and early functional expression of synapse activity translated into increased network activity in $\mathrm{KO}$ cultures. Similar to what we observed in dendrites and synapses, measures of network activity normally observed in more mature WT cultures appeared at much earlier stages of development in neurons developed from $\mathrm{KO}$ clones. Activity was already substantially greater in cultures derived from $\mathrm{KO}$ clones at 2 weeks, a time in development when there is very little activity present in WT cultures. In addition, statistical analysis of network activity that considered time as a factor demonstrated that the trajectory of neuronal activity was distinct in $\mathrm{KO}$ cultures compared with WT controls (Fig. 7). Indeed, activity increased at a much greater rate in $\mathrm{KO}$ cultures, compared with WTs, over the first several weeks of development. Networks formed from iNeurons exhibited bursting behavior as a function of time in vitro, with older cultures exhibiting more robust bursting behavior (Fischer and Gillardon, 2019). Network bursting is driven in part by increased functional synaptic connectivity among neurons (Suresh et al., 2016; Nehme et al., 2018). KO neurons extended dendrites more quickly and had greater numbers of postsynaptic structures. Thus, KO neurons would be expected to exhibit enhanced connectivity at earlier time points compared with control cultures. Increased functional connectivity in $\mathrm{KO}$ networks, driven by longer dendrites with more synaptic structures may contribute to the precocious onset of coordinated network bursting behavior observed in MEA experiments. The effects observed on network activity were apparent at the level of individual clones when grouped by SYNGAP1 genotype. These data further strengthen the conclusion that loss of SynGAP protein drives effects on network activity, and these data provide a possible neurobiological mechanism for why individuals with SYNGAP1 mutations have such a high incidence of early-onset pediatric seizures (Vlaskamp et al., 2019). While
A

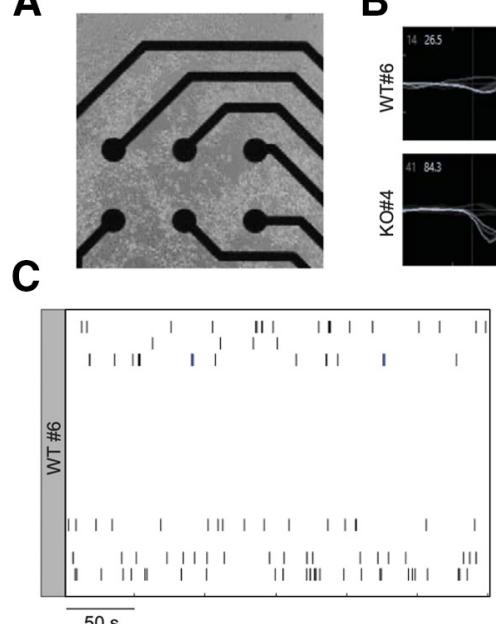

B
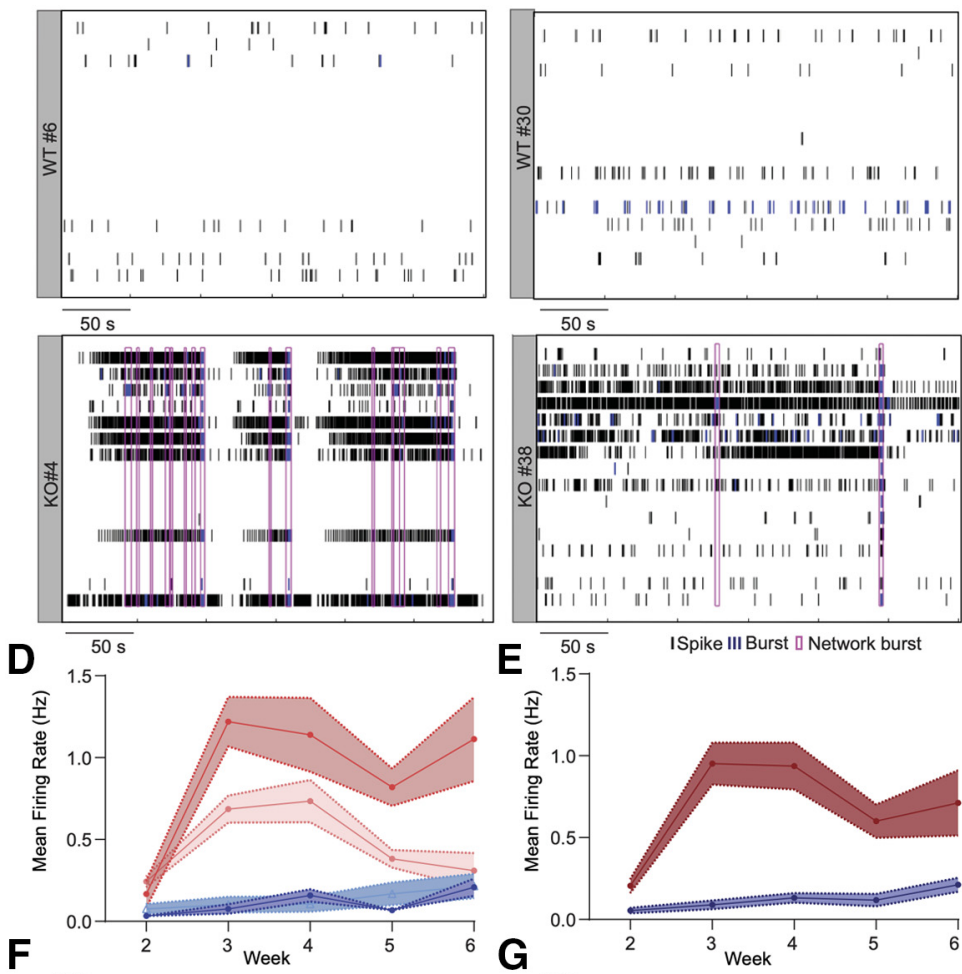

E $\overline{50 \mathrm{~s}} \quad$ ISpike III Burst $\square$ Network burst
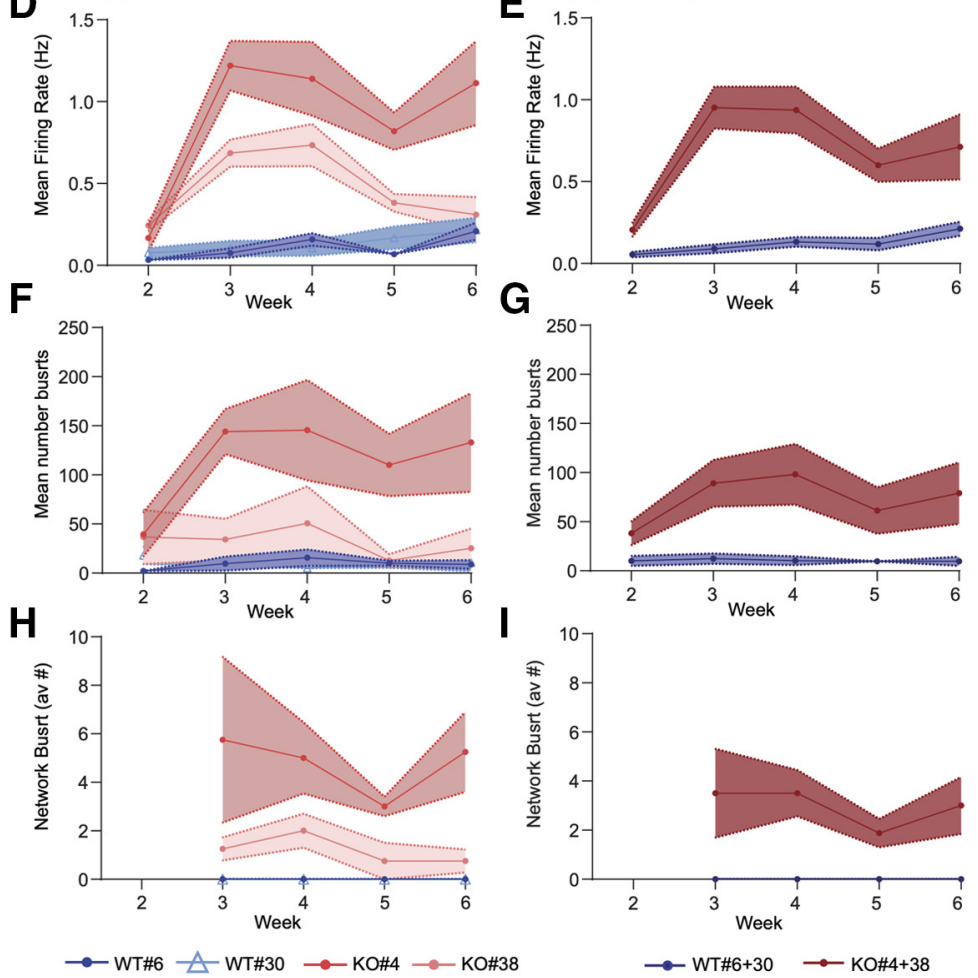

Figure 7. Earlier onset and elevated levels of network activity in SYNGAP1 KO iNeurons. A, Representative bright-field image of 1-week-old iNeurons differentiated from iPSC-derived NPCs plated on a 16-electrode array of an MEA well. Spontaneous action potentials were recorded from the homozygous SYNGAP1 null (KO\#4 and \#38) and control (WT\#6 and \#30) neurons. $\boldsymbol{B}$, Representative waveforms of spiking behavior from a single electrode for each Homo and WT neuronal culture. C, Representative temporal raster plots of KO iNeurons (KO\#4 and \#38) and WT isogenic control iNeurons (WT\#6 and \#30) over 5 min of continuous recording during culture week 3. D, E, Cumulative plots of mean firing rates for all four clones individually $(\boldsymbol{D})$ and grouped together by genotype $(\boldsymbol{E})$, along a developmental timeline. $\boldsymbol{F}, \boldsymbol{G}$, Cumulative plots of average number of bursts for individual clones $(\boldsymbol{F})$ and grouped together by genotype $(\boldsymbol{G})$. $\boldsymbol{H}, \boldsymbol{I}$, Cumulative plots of average number of network bursts for all clone individually $(\boldsymbol{H})$ and grouped together by genotype (I). KO neurons display synaptic connections as early as week 3 of maturation compared with the WT controls. For each clone, four replicates of iNeurons were plated and differentiated concurrently. Graphs represent mean \pm SEM. 
these findings in null iNeurons are intriguing, they must be interpreted with caution with respect to their relevance to disease mechanisms in SYNGAP1 disorders, which are associated with heterozygous variants. First, cellular phenotypes are sometimes, but not always, sensitive to copy number (Yi et al., 2016). As a result, the strong phenotypes present in null iNeurons may be weaker, or nonexistent, in neurons with only one inactivated copy of SYNGAP1. Second, the null models described in this study are based on a single female donor iPSC line. There is no known sex bias in SYNGAP1 disorders (Vlaskamp et al., 2019), although there are reports that sex can generally influence synapse plasticity (Wang et al., 2018; Jain et al., 2019) and more specifically impact how reduced SynGAP expression impacts PSD composition (Mastro et al., 2020). Third, neuronal structure and synapse function studies were performed in a relatively pure glutamatergic iNeuron population produced from Ngn2 expression, whereas MEA studies on network activity were performed in a more complex culture system that used the dualSMAD approach. Because dualSMAD cultures contain additional cell types, including interneurons and glia, the cellular phenotypes described in Ngn2 neurons may not fully explain increased network activity observed in dualSMAD MEA studies. Moreover, Ngn2 iNeurons bypass early NSC-like stages of neuronal development that are associated with ASD pathogenesis (Schafer et al., 2019). These caveats will be addressed in the future by studying iNeurons made from a spectrum of patient-derived iPSCs of different sexes. These future studies should also include more sophisticated cell-based studies in dualSMAD-produced cultures to better define the cellular mechanisms that lead to enhanced network activity in response to SYNGAP1 loss-of-function.

Data implicating SYNGAP1 expression on the structural and functional maturation of human neurons are consistent with known functions of this gene discovered from experimentation in mouse neurons (Kilinc et al., 2018). SynGAP protein is highly expressed in rodent neurons and is capable of bidirectional regulation of excitatory synapse strength. Overexpression of SynGAP protein suppresses excitatory synapse transmission by activating AMPA receptor internalization (Rumbaugh et al., 2006). One report indicates that SynGAP isoforms regulate synaptic strength in opposing directions (McMahon et al., 2012). However, genetic ablation of all Syngap1 splice forms in mice, which removes expression of all protein isoforms, leads to increased excitatory synapse strength and early appearance of synaptic activity in glutamatergic neurons (Clement et al., 2012). These data indicate that the integrated function of all SynGAP proteins in developing mouse neurons is to suppress excitatory synapse function during development. Our findings in human neurons, which also ablated expression of human SynGAP isoforms, support this model of developmental SYNGAP1 function. Given that we observed early and enhanced excitatory synapse function in $\mathrm{KO}$ iNeurons, human SYNGAP1 also appears to slow the onset of excitatory synapse activity by suppressing excitatory synapse function.

The impact of SYNGAP1 on human neuron dendritic maturation is also consistent with observations in rodent neurons. The effect of SynGAP protein expression on rodent neuron dendritic development is complex and depends on the type of neuron and brain area studied. Syngap1 heterozygous KO mice have well-documented impairments in dendritic morphogenesis, which is linked to alterations in neural circuit assembly and neuronal connectivity. Layer 5 (L5) neurons in the somatosensory cortex of these mutant mice undergo a form of accelerated postmitotic differentiation, where dendritic extension proceeds at a quicker pace compared with WT mice (Aceti et al., 2015). Interestingly, these neurons also undergo premature spine morphogenesis and early spine pruning. These observations, combined with a desynchronization of L5 cell body and dendritic arbor growth, strongly indicate that SynGAP expression acts in these neurons to suppress a differentiation program that stimulates neuronal maturation. In contrast to these findings, neurons in the upper lamina (layers 2-4) of the somatosensory cortex of Syngap1 KO mice show the opposite phenotype. These neurons undergo a form of arrested development where dendritic arbors are shorter compared with similar neurons in WT littermates (Michaelson et al., 2018). Neurons with shorter dendritic arbors also had fewer dendritic spines, and these structural alterations impacted connectivity within somatosensory cortex circuits. While our studies in human iNeurons support a role for SYNGAP1 to suppress dendritic maturation, the specific effect of the gene on structural maturation may also be dependent on the type of human neuron. Two-dimensional neuronal cultures lack the cellular complexity of neural networks found in the intact nervous system. It will be of considerable interest to assess how loss of SYNGAP1 expression impacts various types of genetically and morphologically distinct neurons formed in three-dimensional human culture systems, such as organoids, and how alterations to dendritic morphogenesis may contribute to impaired neural circuit connectivity and development of network activity.

\section{References}

Aceti M, Creson TK, Vaissiere T, Rojas C, Huang WC, Wang YX, Petralia RS, Page DT, Miller CA, Rumbaugh G (2015) Syngap1 haploinsufficiency damages a postnatal critical period of pyramidal cell structural maturation linked to cortical circuit assembly. Biol Psychiatry 77:805-815.

Berryer MH, Hamdan FF, Klitten LL, Møller RS, Carmant L, Schwartzentruber J, Patry L, Dobrzeniecka S, Rochefort D, NeugnotCerioli M, Lacaille JC, Niu Z, Eng CM, Yang Y, Palardy S, Belhumeur C, Rouleau GA, Tommerup N, Immken L, Beauchamp MH, et al. (2013) Mutations in SYNGAP1 cause intellectual disability, autism, and a specific form of epilepsy by inducing haploinsufficiency. Hum Mutat 34:385-394.

Carvill GL, Heavin SB, Yendle SC, McMahon JM, O'Roak BJ, Cook J, Khan A, Dorschner MO, Weaver M, Calvert S, Malone S, Wallace G, Stanley T, Bye AM, Bleasel A, Howell KB, Kivity S, Mackay MT, Rodriguez-Casero V, Webster R, et al. (2013) Targeted resequencing in epileptic encephalopathies identifies de novo mutations in CHD2 and SYNGAP1. Nat Genet 45:825-830.

Charrier C, Joshi K, Coutinho-Budd J, Kim JE, Lambert N, de Marchena J, Jin WL, Vanderhaeghen P, Ghosh A, Sassa T, Polleux F (2012) Inhibition of SRGAP2 function by its human-specific paralogs induces neoteny during spine maturation. Cell 149:923-935

Clement JP, Aceti M, Creson TK, Ozkan ED, Shi Y, Reish NJ, Almonte AG, Miller BH, Wiltgen BJ, Miller CA, Xu X, Rumbaugh G (2012) Pathogenic SYNGAP1 mutations impair cognitive development by disrupting maturation of dendritic spine synapses. Cell 151:709-723.

Clement JP, Ozkan ED, Aceti M, Miller CA, Rumbaugh G (2013) SYNGAP1 links the maturation rate of excitatory synapses to the duration of critical-period synaptic plasticity. J Neurosci 33:10447-10452.

Deciphering Developmental Disorders Study (2015) Large-scale discovery of novel genetic causes of developmental disorders. Nature 519:223-228.

Deciphering Developmental Disorders Study (2017) Prevalence and architecture of de novo mutations in developmental disorders. Nature 542:433-438.

Fischer SK, Gillardon F (2019) Multielectrode array recordings of human iPSC-derived neurons reveal differences in network activity depending on differentiation protocol and genome modification. Conference Abstract: MEA Meeting 2018 | 11th International Meeting on Substrate Integrated Microelectrode Arrays.

Gamache TR, Araki Y, Huganir RL (2020) Twenty years of SynGAP research: from synapses to cognition. J Neurosci 40:1596-1605.

Gou G, Roca-Fernandez A, Kilinc M, Serrano E, Reig-Viader R, Araki Y, Huganir RL, de Quintana-Schmidt C, Rumbaugh G, Bayés À (2020) SynGAP splice variants display heterogeneous spatio-temporal expression and subcellular distribution in the developing mammalian brain. J 
Neurochem. Advance online publication. Retrieved Feb 18, 2020. doi: 10.1111/jnc. 14988 .

Hamdan FF, Gauthier J, Spiegelman D, Noreau A, Yang Y, Pellerin S, Dobrzeniecka S, Côté M, Perreau-Linck E, Perreault-Linck E, Carmant L, D'Anjou G, Fombonne E, Addington AM, Rapoport JL, Delisi LE, Krebs MO, Mouaffak F, Joober R, Mottron L, et al. (2009) Mutations in SYNGAP1 in autosomal nonsyndromic mental retardation. N Engl J Med 360:599-605.

Hamdan FF, Daoud H, Piton A, Gauthier J, Dobrzeniecka S, Krebs MO, Joober R, Lacaille JC, Nadeau A, Milunsky JM, Wang Z, Carmant L, Mottron L, Beauchamp MH, Rouleau GA, Michaud JL (2011) De novo SYNGAP1 mutations in nonsyndromic intellectual disability and autism. Biol Psychiatry 69:898-901.

Jain A, Huang GZ, Woolley CS (2019) Latent sex differences in molecular signaling that underlies excitatory synaptic potentiation in the hippocampus. J Neurosci 39:1552-1565.

Jiang X, Chen J, Bajić A, Zhang C, Song X, Carroll SL, Cai ZL, Tang M, Xue M, Cheng N, Schaaf CP, Li F, MacKenzie KR, Ferreon AC, Xia F, Wang MC, Maletić-Savatić M, Wang J (2017) Quantitative real-time imaging of glutathione. Nat Commun 8:16087.

Jimenez-Gomez A, Niu S, Andujar-Perez F, McQuade EA, Balasa A, Huss D, Coorg R, Quach M, Vinson S, Risen S, Holder JL Jr (2019) Phenotypic characterization of individuals with SYNGAP1 pathogenic variants reveals a potential correlation between posterior dominant rhythm and developmental progression. J Neurodev Disord 11:18.

Karczewski KJ, Francioli LC, Tiao G, Cummings BB, Alföldi J, Wang Q, Collins RL, Laricchia KM, Ganna A, Birnbaum DP, Gauthier LD, Brand H, Solomonson M, Watts NA, Rhodes D, Singer-Berk M, England EM, Seaby EG, Kosmicki JA, Walters RK, et al. (2020) The mutational constraint spectrum quantified from variation in 141,456 humans. Nature 581:434-443.

Kilinc M, Creson T, Rojas C, Aceti M, Ellegood J, Vaissiere T, Lerch JP, Rumbaugh G (2018) Species-conserved SYNGAP1 phenotypes associated with neurodevelopmental disorders. Mol Cell Neurosci 91:140-150.

Kim JH, Lee HK, Takamiya K, Huganir RL (2003) The role of synaptic GTPase-activating protein in neuronal development and synaptic plasticity. J Neurosci 23:1119-1124.

Komiyama NH, Watabe AM, Carlisle HJ, Porter K, Charlesworth P, Monti J, Strathdee DJ, O'Carroll CM, Martin SJ, Morris RG, O'Dell TJ, Grant SG (2002) SynGAP regulates ERK/MAPK signaling, synaptic plasticity, and learning in the complex with postsynaptic density 95 and NMDA receptor. J Neurosci 22:9721-9732.

Mastro TL, Preza A, Basu S, Chattarji S, Till SM, Kind PC, Kennedy MB (2020) A sex difference in the response of the rodent postsynaptic density to synGAP haploinsufficiency. eLife 9:e52656.

McMahon AC, Barnett MW, O'Leary TS, Stoney PN, Collins MO, Papadia S, Choudhary JS, Komiyama NH, Grant SG, Hardingham GE, Wyllie DJ, Kind PC (2012) SynGAP isoforms exert opposing effects on synaptic strength. Nat Commun 3:900.

Michaelson SD, Ozkan ED, Aceti M, Maity S, Llamosas N, Weldon M, Mizrachi E, Vaissiere T, Gaffield MA, Christie JM, Holder JL Jr, Miller CA, Rumbaugh G (2018) SYNGAP1 heterozygosity disrupts sensory processing by reducing touch-related activity within somatosensory cortex circuits. Nat Neurosci 21:1-13.

Nehme R, Zuccaro E, Ghosh SD, Li C, Sherwood JL, Pietilainen O, Barrett LE, Limone F, Worringer KA, Kommineni S, Zang Y, Cacchiarelli D, Meissner A, Adolfsson R, Haggarty S, Madison J, Muller M, Arlotta P, Fu Z, Feng G, et al. (2018) Combining NGN2 programming with developmental patterning generates human excitatory neurons with NMDARmediated synaptic transmission. Cell Rep 23:2509-2523.

O'Roak BJ, Stessman HA, Boyle EA, Witherspoon KT, Martin B, Lee C, Vives L, Baker C, Hiatt JB, Nickerson DA, Bernier R, Shendure J, Eichler
EE (2014) Recurrent de novo mutations implicate novel genes underlying simplex autism risk. Nat Commun 5:5595.

Ozkan ED, Creson TK, Kramar EA, Rojas C, Seese RR, Babyan AH, Shi Y, Lucero R, Xu X, Noebels JL, Miller CA, Lynch G, Rumbaugh G (2014) Reduced cognition in Syngap1 mutants is caused by isolated damage within developing forebrain excitatory neurons. Neuron 82:1317-1333.

Parker MJ, Fryer AE, Shears DJ, Lachlan KL, McKee SA, Magee AC, Mohammed S, Vasudevan PC, Park SM, Benoit V, Lederer D, Maystadt I, Study D, FitzPatrick DR (2015) De novo, heterozygous, loss-of-function mutations in SYNGAP1 cause a syndromic form of intellectual disability. Am J Med Genet A 167A:2231-2237.

Petanjek Z, Judas M, Simic G, Rasin MR, Uylings HB, Rakic P, Kostovic I (2011) Extraordinary neoteny of synaptic spines in the human prefrontal cortex. Proc Natl Acad Sci USA 108:13281-13286.

Rauch A, Wieczorek D, Graf E, Wieland T, Endele S, Schwarzmayr T, Albrecht B, Bartholdi D, Beygo J, Di Donato N, Dufke A, Cremer K, Hempel M, Horn D, Hoyer J, Joset P, Röpke A, Moog U, Riess A, Thiel CT, et al. (2012) Range of genetic mutations associated with severe nonsyndromic sporadic intellectual disability: an exome sequencing study. Lancet 380:1674-1682.

Rumbaugh G, Adams JP, Kim JH, Huganir RL (2006) SynGAP regulates synaptic strength and mitogen-activated protein kinases in cultured neurons. Proc Natl Acad Sci USA 103:4344-4351.

Satterstrom FK, Kosmicki JA, Wang J, Breen MS, De Rubeis S, An JY, Peng M, Collins R, Grove J, Klei L, Stevens C, Reichert J, Mulhern MS, Artomov M, Gerges S, Sheppard B, Xu X, Bhaduri A, Norman U, Brand $\mathrm{H}$, et al. (2020) Large-scale exome sequencing study implicates both developmental and functional changes in the neurobiology of autism. Cell 180:568-584.e23.

Schafer ST, Paquola AC, Stern S, Gosselin D, Ku M, Pena M, Kuret TJ, Liyanage M, Mansour AA, Jaeger BN, Marchetto MC, Glass CK, Mertens J, Gage FH (2019) Pathological priming causes developmental gene network heterochronicity in autistic subject-derived neurons. Nat Neurosci 22:243-255.

Sridharan B, Hubbs C, Llamosas N, Kilinc M, Singhera FU, Willems E, Piper DR, Scampavia L, Rumbaugh G, Spicer TP (2019) A simple procedure for creating scalable phenotypic screening assays in human neurons. Sci Rep 9:9000

Suresh J, Radojicic M, Pesce LL, Bhansali A, Wang J, Tryba AK, Marks JD, van Drongelen W (2016) Network burst activity in hippocampal neuronal cultures: the role of synaptic and intrinsic currents. J Neurophysiol 115:3073-3089.

Vlaskamp DR, Shaw BJ, Burgess R, Mei D, Montomoli M, Xie H, Myers CT, Bennett MF, XiangWei W, Williams D, Maas SM, Brooks AS, Mancini GM, van de Laar IM, van Hagen JM, Ware TL, Webster RI, Malone S, Berkovic SF, Kalnins RM, et al. (2019) SYNGAP1 encephalopathy: a distinctive generalized developmental and epileptic encephalopathy. Neurology 92:e96-e107.

von Stulpnagel C, Funke C, Haberl C, Hortnagel K, Jungling J, Weber YG, Staudt M, Kluger G (2015) SYNGAP1 mutation in focal and generalized epilepsy: a literature overview and a case report with special aspects of the EEG. Neuropediatrics 46:287-291.

Wang W, Le AA, Hou B, Lauterborn JC, Cox CD, Levin ER, Lynch G, Gall CM (2018) Memory-related synaptic plasticity is sexually dimorphic in rodent hippocampus. J Neurosci 38:7935-7951.

Yi F, Danko T, Botelho SC, Patzke C, Pak C, Wernig M, Sudhof TC (2016) Autism-associated SHANK3 haploinsufficiency causes Ih channelopathy in human neurons. Science 352:aaf2669.

Zhang Y, Pak C, Han Y, Ahlenius H, Zhang Z, Chanda S, Marro S, Patzke C, Acuna C, Covy J, Xu W, Yang N, Danko T, Chen L, Wernig M, Sudhof TC (2013) Rapid single-step induction of functional neurons from human pluripotent stem cells. Neuron 78:785-798. 